\title{
THE STRUCTURE OF TAME MINIMAL DYNAMICAL SYSTEMS FOR GENERAL GROUPS
}

\author{
ELI GLASNER
}

\begin{abstract}
We use the structure theory of minimal dynamical systems to show that, for a general group $\Gamma$, a tame, metric, minimal dynamical system $(X, \Gamma)$ has
\end{abstract} the following structure:

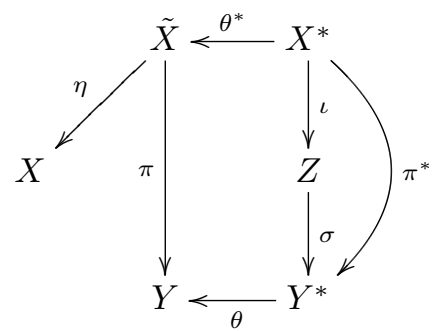

Here (i) $\tilde{X}$ is a metric minimal and tame system (ii) $\eta$ is a strongly proximal extension, (iii) $Y$ is a strongly proximal system, (iv) $\pi$ is a point distal and RIM extension with unique section, (v) $\theta, \theta^{*}$ and $\iota$ are almost one-to-one extensions, and (vi) $\sigma$ is an isometric extension.

When the map $\pi$ is also open this diagram reduces to

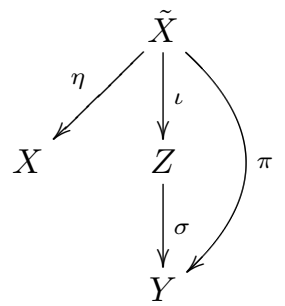

In general the presence of the strongly proximal extension $\eta$ is unavoidable. If the system $(X, \Gamma)$ admits an invariant measure $\mu$ then $Y$ is trivial and $X=\tilde{X}$ is an almost automorphic system; i.e. $X \stackrel{\iota}{\rightarrow} Z$, where $\iota$ is an almost one-to-one extension and $Z$ is equicontinuous. Moreover, $\mu$ is unique and $\iota$ is a measure theoretical isomorphism $\iota:(X, \mu, \Gamma) \rightarrow(Z, \lambda, \Gamma)$, with $\lambda$ the Haar measure on $Z$. Thus, this is always the case when $\Gamma$ is amenable.

\section{Contents}

Introduction

1. A brief survey of abstract topological dynamics

2. On semiopen maps

Date: June, 2016.

Key words and phrases. Enveloping semigroup, tame system, strong proximality, almost automorphic system, relatively invariant measure (RIM).

2000 Mathematical Subject Classification 54H20.

This research was supported by a grant of the Israel Science Foundation (ISF 668/13). 
3. A key proposition on diffused measures 13

4. Some properties of tame minimal systems 16

5. The structure of tame metric minimal dynamical systems 18

6. Examples 23

7. Appendix: Borel's density theorem 24

References $\quad 25$

\section{INTRODUCTION}

In this work we prove a structure theorem for tame, metric, minimal dynamical systems for an arbitrary acting group, Theorem 5.3 and Corollary 5.4. This generalizes an older result which delt with the case of abelian acting group ([26], [28] and [21]). It turns out that the key property needed for the simple form of the structure theorem is amenability, rather than commutativity. In particular Corollary 5.4 asserts that, when the acting group is amenable, every metric minimal tame system is almost automorphic. When the group is not amenable the structure of a minimal tame system is more complicated and may involve a nontrivial strongly proximal factor.

The method of proof we use is basically the same one we applied in [21] and consists mainly in applying various structure theorems for general minimal systems. After some efforts to write our proof as a commentary on [21] with indications where new ideas and results appear, we found that the best way (for the reader as well as for the author) to present the new results is to start anew, although for some minor points we nevertheless refer to the older work. For completeness' sake and in order to provide the reader with the appropriate background I also reproduce the review section, with the necessary changes.

A dynamical system is a pair $(X, \Gamma)$, where $X$ is a compact Hausdorff space and $\Gamma$ an infinite abstract group acting as a group of homeomorphisms of the space $X$. That is, we are given a homomorphism (not necessarily an isomorphism) of $\Gamma$ into Homeo (X). For $\gamma \in \Gamma$ and $x \in X$ we write $\gamma x$ for the image of $x$ under the homeomorphism which corresponds to $\gamma$. We will often abuse this notation and consider $\gamma$ as a homeomorphism of $X$.

The enveloping semigroup $E(X, \Gamma)$ of the dynamical system $(X, \Gamma)$ is defined as the closure of image of $\Gamma$ in the product space $X^{X}$. It is not hard to check that, under composition of maps, $E(X, \Gamma)$ is a compact right topological semigroup, i.e. for each $q \in E(X, \Gamma)$ the map $R_{q}: p \mapsto p q$ is continuous. In fact the canonical homomorphism of $\Gamma$ into $E(X, \Gamma)$ is a right topological semigroup compactification of $\Gamma$; i.e. it has a dense range and for each $\gamma \in \Gamma$ multiplication on the left $L_{\gamma}: p \mapsto \gamma p$ is continuous on $E(X, \Gamma)$. This left multiplication by elements of $\Gamma$ makes $(E(X, \Gamma), \Gamma)$ a dynamical system.

The enveloping semigroup was introduced by Robert Ellis in 1960 and became an indispensable tool in abstract topological dynamics. However explicit computations of enveloping semigroups are quite rare. One reason for this is that often $E(X, \Gamma)$ is non-metrizable. 
Following a pioneering work of A. Köller, [29], Glasner and Megrelishvily proved the following dynamical version of the Bourgain-Fremlin-Talagrand dichotomy theorem, [20], [22].

0.1. Theorem (A dynamical BFT dichotomy). Let $(X, \Gamma)$ be a metric dynamical system and let $E(X, \Gamma)$ be its enveloping semigroup. We have the following dichotomy. Either

1. $E(X, \Gamma)$ is a separable Fréchet compact space, hence with cardinality card $E(X, \Gamma) \leq$ $2^{\aleph_{0}} ;$ or

2. the compact space $E$ contains a homeomorphic copy of $\beta \mathbb{N}$, hence card $E(X, \Gamma)=$ $2^{2^{\aleph_{0}}}$.

A dynamical system is called tame if the first alternative occurs, i.e. $E(X, \Gamma)$ is Fréchet. A dynamical characterization of tame metrizable dynamical systems follows, [22] and [25]:

0.2. Theorem. A compact metric dynamical system $(X, \Gamma)$ is tame if and only if every element of $E(X, \Gamma)$ is a Baire class 1 function from $X$ to itself.

For the definitions of HAE (hereditarily almost equicontinuous) systems and other undefined notions which appear in the following theorem, as well as for some further motivation and examples we refer the reader to the papers [22], [20] and [25].

0.3. Theorem ([25]). Let $X$ be a compact metric $G$-space. The following conditions are equivalent:

1. the dynamical system $(X, \Gamma)$ is hereditarily almost equicontinuous (HAE);

2. the dynamical system $(X, \Gamma)$ is $R N$, that is, it admits a proper representation on a Radon-Nikodym Banach space;

3. the enveloping semigroup $E(X, \Gamma)$ is metrizable.

It thus follows that every metric HAE system is tame.

I would like to thank the two anonymous referees for their corrections and helpful suggestions.

\section{A BRIEF SURVEY OF ABSTRACT TOPOLOGICAL DYNAMICS}

This section is a brief review of the structure theory of minimal dynamical systems. We will emphasize some aspects which will be relevant in the present work. For full details the reader is referred to the books [10], [16], [2] and [34] and the review articles [33] and [19].

If $X$ is a topological space and $A \subset X$ then we use $\bar{A}=\operatorname{cls} \mathrm{A}, A^{\circ}, \partial A$ and $A^{c}=X \backslash A$ for the closure, interior, boundary and complementation operations on $A$, respectively.

A topological dynamical system or briefly a system is a pair $(X, \Gamma)$, where $X$ is a compact Hausdorff space and $\Gamma$ an abstract infinite group which acts on $X$ as a group of homeomorphisms. When there is no room for confusion we write $X$ for the system $(X, \Gamma)$. A sub-system of $(X, \Gamma)$ is a closed invariant subset $Y \subset X$ with the restricted action. For a point $x \in X$, we let $\mathcal{O}_{\Gamma}(x)=\{\gamma x: \gamma \in \Gamma\}$, and $\overline{\mathcal{O}}_{\Gamma}(x)=\operatorname{cls}\{\gamma x: \gamma \in \Gamma\}$. These subsets of $X$ are called the orbit and orbit closure of $x$ respectively. We say 
that $(X, \Gamma)$ is point transitive if there exists a point $x \in X$ with a dense orbit. In that case $x$ is called a transitive point. If every point is transitive we say that $(X, \Gamma)$ is a minimal system. We say that $x \in X$ is an almost periodic or a minimal point if $\overline{\mathcal{O}}_{\Gamma}(x)$ is a minimal system.

The dynamical system $(X, \Gamma)$ is topologically transitive if for any two nonempty open subsets $U$ and $V$ of $X$ there exists some $\gamma \in \Gamma$ with $\gamma U \cap V \neq \emptyset$. Clearly a point transitive system is topologically transitive and when $X$ is metrizable the converse holds as well: in a metrizable topologically transitive system the set of transitive points is a dense $G_{\delta}$ subset of $X$.

The system $(X, \Gamma)$ is weakly mixing if the product system $(X \times X, \Gamma)$ (where $\left.\gamma\left(x, x^{\prime}\right)=\left(\gamma x, \gamma x^{\prime}\right), x, x^{\prime} \in X, \gamma \in \Gamma\right)$ is topologically transitive.

If $(Y, \Gamma)$ is another system then a continuous onto map $\pi: X \rightarrow Y$ satisfying $\gamma \circ \pi=\pi \circ \gamma$ for every $\gamma \in \Gamma$ is called a homomorphism of dynamical systems. In this case we say that $(Y, \Gamma)$ is a factor of $(X, \Gamma)$ and also that $(X, \Gamma)$ is an extension of $(Y, \Gamma)$. With the system $(X, \Gamma)$ we associate the induced action (the hyper system associated with $(X, \Gamma))$ on the compact space $2^{X}$ of closed subsets of $X$ equipped with the Vietoris topology. A subsystem $Y$ of $\left(2^{X}, \Gamma\right)$ is a quasifactor of $(X, \Gamma)$ if $\bigcup\{A: A \in Y\}=X$.

The system $(X, \Gamma)$ can always be considered as a quasifactor of $(X, \Gamma)$ by identifying $x$ with $\{x\}$. Recall that if $(X, \Gamma) \stackrel{\pi}{\rightarrow}(Y, \Gamma)$ is a homomorphism then in general $\pi^{-1}: Y \rightarrow 2^{X}$ is an upper-semi-continuous map and that $\pi: X \rightarrow Y$ is open iff $\pi^{-1}: Y \rightarrow 2^{X}$ is continuous.

We assume for simplicity that our acting group $\Gamma$ is a discrete group. $\beta \Gamma$ will denote the Stone-Čech compactification of $\Gamma$. The universal properties of $\beta \Gamma$ make it

- a compact semigroup with right continuous multiplication (for a fixed $p \in \beta \Gamma$ the map $q \mapsto q p, q \in \beta \Gamma$ is continuous), and left continuous multiplication by elements of $\Gamma$, considered as elements of $\beta \Gamma$ (for a fixed $\gamma \in \Gamma$ the map $q \mapsto \gamma q, q \in \beta \Gamma$ is continuous $)$.

- a dynamical system $(\beta \Gamma, \Gamma)$ under left multiplication by elements of $\Gamma$.

The system $(\beta \Gamma, \Gamma)$ is the universal point transitive $\Gamma$-system; i.e. for every point transitive system $(X, \Gamma)$ and a point $x \in X$ with dense orbit, there exists a homomorphism of systems $(\beta \Gamma, \Gamma) \rightarrow(X, \Gamma)$ which sends $e$, the identity element of $\Gamma$, onto $x$. For $p \in \beta \Gamma$ we let $p x$ denote the image of $p$ under this homomorphism. This defines an "action" of the semigroup $\beta \Gamma$ on every dynamical system. In fact, by universality there exists a unique homomorphism $(\beta \Gamma, \Gamma) \rightarrow(E(X, \Gamma), \Gamma)$ onto the enveloping semigroup $E(X, \Gamma)$ which is also a semigroup homomorphism and we can interpret, and often do, the $\beta \Gamma$ action on $X$ via this homomorphism.

When dealing with the hyper system $\left(2^{X}, \Gamma\right)$ we write $p \circ A$ for the image of the closed subset $A \subset X$ under $p \in \beta \Gamma$ to distinguish it from the (usually non-closed) subset $p A=\{p x: x \in A\}$. If $p$ is the limit of a net $\gamma_{i}$ in $\Gamma$ then

$$
p \circ A=\left\{x \in X: \text { there are a subnet } \gamma_{i_{j}} \text { and a net } x_{j} \in A \text { with } x=\lim _{j} \gamma_{i_{j}} x_{j}\right\} \text {. }
$$

We always have $p A \subset p \circ A$. 
The compact semigroup $\beta \Gamma$ has a rich algebraic structure. For instance for countable $\Gamma$ there are $2^{c}$ minimal left (necessarily closed) ideals in $\beta \Gamma$ all isomorphic as systems and each serving as a universal minimal system. Each such minimal ideal, say $M$, has a subset $J$ of $2^{c}$ idempotents such that $\{v M: v \in J\}$ is a partition of $M$ into disjoint isomorphic (non-closed) subgroups. An idempotent in $\beta \Gamma$ is called minimal if it belongs to some minimal ideal. A point $x$ in a dynamical system $(X, \Gamma)$ is a minimal point iff there is some minimal idempotent $v$ in $\beta \Gamma$ with $v x=x$, iff there exists some $v \in J$ with $v x=x$.

The group of dynamical system automorphisms of $(M, \Gamma), G=\operatorname{Aut}(M, \Gamma)$ can be identified with any one of the groups $v M$ as follows: with $\alpha \in v M$ we associate the automorphism $\hat{\alpha}:(M, \Gamma) \rightarrow(M, \Gamma)$ given by right multiplication $\hat{\alpha}(p)=p \alpha, p \in M$. The group $G$ plays a central role in the algebraic theory. It carries a natural $T_{1}$ compact topology, called by Ellis the $\tau$-topology, which is weaker than the relative topology induced on $G=u M$ as a subset of $M$. The $\tau$-closure of a subset $A$ of $G$ consists of those $\beta \in G$ for which the set $\operatorname{graph}(\beta)=\{(p, p \beta): p \in M\}$ is a subset of the closure in $M \times M$ of the set $\bigcup\{$ graph $(\alpha): \alpha \in A\}$. Both right and left multiplication on $G$ are $\tau$ continuous and so is inversion.

It is convenient to fix a minimal left ideal $M$ in $\beta \Gamma$ and an idempotent $u \in M$. As explained above we identify $G$ with $u M$ and it follows that for any subset $A \subset G$,

$$
\operatorname{cls}_{\tau} A=u(u \circ A)=G \cap(u \circ A) .
$$

Also in this way we can consider the "action" of $G$ on every system $(X, \Gamma)$ via the action of $\beta \Gamma$ on $X$. With every minimal system $(X, \Gamma)$ and a point $x_{0} \in u X=\{x \in$ $X: u x=x\}$ we associate a $\tau$-closed subgroup

$$
\mathfrak{G}\left(X, x_{0}\right)=\left\{\alpha \in G: \alpha x_{0}=x_{0}\right\}
$$

the Ellis group of the pointed system $\left(X, x_{0}\right)$. The quotient space $G / \mathfrak{G}\left(X, x_{0}\right)$ can be identified with the subset $u X \subset X$ via the map $\alpha \mapsto \alpha x_{0}$ and the induced quotient $\tau$-topology is called the $\tau$-topology on $u X$. Again the $\tau$-topology is weaker than the relative topology induced on $u X$ as a subset of $X$, it is $T_{1}$ and compact, and the closure operation is given by

$$
\operatorname{cls}_{\tau} A=u(u \circ A)=u X \cap(u \circ A), \quad A \subset u X .
$$

For a homomorphism $\pi: X \rightarrow Y$ with $\pi\left(x_{0}\right)=y_{0}$ we have

$$
\mathfrak{G}\left(X, x_{0}\right) \subset \mathfrak{G}\left(Y, y_{0}\right) .
$$

For a $\tau$-closed subgroup $F$ of $G$ the derived group $F^{\prime}$ is given by:

$$
F^{\prime}:=\bigcap\left\{\operatorname{cls}_{\tau} O: O \text { a } \tau \text {-open neighborhood of } u \text { in } F\right\} .
$$

$F^{\prime}$ is a $\tau$-closed normal (in fact characteristic) subgroup of $F$ and it is characterized as the smallest $\tau$-closed subgroup $H$ of $F$ such that $F / H$ is a compact Hausdorff topological group. In particular, for an abelian $\Gamma$, the topological group $G / G^{\prime}$ is the Bohr compactification of $\Gamma$.

A pair of points $\left(x, x^{\prime}\right) \in X \times X$ for a system $(X, \Gamma)$ is called proximal if there exists a net $\gamma_{i} \in \Gamma$ and a point $z \in X$ such that $\lim \gamma_{i} x=\lim \gamma_{i} x^{\prime}=z$ (iff there exists 
$p \in \beta \Gamma$ with $p x=p x^{\prime}$. We denote by $P$ the set of proximal pairs in $X \times X$. We have

$$
P=\bigcap\{\Gamma V: V \text { a neighborhood of the diagonal in } X \times X\} .
$$

We write $P[x]:=\left\{x^{\prime} \in X:\left(x, x^{\prime}\right) \in P\right\}$. A system $(X, \Gamma)$ is called proximal when $P=X \times X$ and distal when $P=\Delta$, the diagonal in $X \times X$. It is called strongly proximal when the following much stronger condition holds: the dynamical system $(\mathfrak{M}(X), \Gamma)$, induced on the compact space $\mathfrak{M}(X)$ of Borel probability measures on $X$, is proximal. A minimal system $(X, \Gamma)$ is called point distal if there exists a point $x \in X$ such that if $x, x^{\prime}$ is a proximal pair then $x=x^{\prime}$.

The regionally proximal relation on $X$ is defined by

$$
Q=\bigcap\{\overline{\Gamma V}: V \text { a neighborhood of } \Delta \text { in } X \times X\}
$$

It is easy to verify that $Q$ is trivial - i.e. equals $\Delta$ - iff the system is equicontinuous.

An extension $(X, \Gamma) \stackrel{\pi}{\rightarrow}(Y, \Gamma)$ of minimal systems is called a proximal extension if the relation $R_{\pi}=\left\{\left(x, x^{\prime}\right): \pi(x)=\pi\left(x^{\prime}\right)\right\}$ satisfies $R_{\pi} \subset P$, and a distal extension when $R_{\pi} \cap P=\Delta$. One can show that every distal extension is open. $\pi$ is a highly proximal (HP) extension if for every closed subset $A$ of $X$ with $\pi(A)=Y$, necessarily $A=X$. It is easy to see that a HP extension is proximal. In the metric case an extension $(X, \Gamma) \stackrel{\pi}{\rightarrow}(Y, \Gamma)$ of minimal systems is HP iff it is an almost 1-1 extension, that is the set $\left\{x \in X: \pi^{-1}(\pi(x))=\{x\}\right.$ is a dense $G_{\delta}$ subset of $X$.

The map $\pi$ is strongly proximal if for every $y \in Y$ and every probability measure $\nu$ with $\operatorname{supp} \nu \subset \pi^{-1}(y)$, there exists a net $\gamma_{i} \in \Gamma$ and a point $x \in X$ such that $\lim _{i} \gamma_{i} \nu=\delta_{x}$ in the weak* topology on the space $\mathfrak{M}(X)$ of probability measures on $X$.

The extension $\pi$ is called an equicontinuous extension if for every $\epsilon$, a neighborhood of the diagonal $\Delta=\{(x, x): x \in X\} \subset X \times X$, there exists a neighborhood of the diagonal $\delta$ such that $\gamma\left(\delta \cap R_{\pi}\right) \subset \epsilon$ for every $\gamma \in \Gamma$. In the metric case an equicontinuous extension is also called an isometric extension.

A minimal dynamical system $(X, \Gamma)$ is called almost automorphic if it has the form $X \stackrel{\theta}{\rightarrow} Z$, where $Z$ is equicontinuous and $\theta$ is almost one-to-one. More generally, an extension of minimal systems $\pi: X \rightarrow Y$ is called an almost automorphic extension if it has the form $X \stackrel{\theta}{\rightarrow} Z \stackrel{\sigma}{\rightarrow} Y$, where $\theta$ is an almost one-to-one extension, $\sigma$ is an isometric extension and $\pi=\sigma \circ \theta$.

The extension $\pi$ is a weakly mixing extension when $R_{\pi}$ as a subsystem of the product system $(X \times X, \Gamma)$ is topologically transitive.

The algebraic language is particularly suitable for dealing with such notions. For example an extension $(X, \Gamma) \stackrel{\pi}{\rightarrow}(Y, \Gamma)$ of minimal systems is a proximal extension iff the Ellis groups $\mathfrak{G}\left(X, x_{0}\right)=A$ and $\mathfrak{G}\left(Y, y_{0}\right)=F$ coincide. It is distal iff for every $y \in Y$, and $x \in \pi^{-1}(y), \pi^{-1}(y)=\mathfrak{G}(Y, y) x ;$ iff:

for every $y=p y_{0} \in Y, p$ an element of $M, \pi^{-1}(y)=p \pi^{-1}\left(y_{0}\right)=p F x_{0}$, where $F=\mathfrak{G}\left(Y, y_{0}\right)$.

In particular $(X, \Gamma)$ is distal iff $G x=X$ for some (hence every) $x \in X$. The extension $\pi$ is an equicontinuous extension iff it is a distal extension and, denoting $\mathfrak{G}\left(X, x_{0}\right)=A$ 
and $\mathfrak{G}\left(Y, y_{0}\right)=F$

$$
F^{\prime} \subset A
$$

In this case, setting $A_{0}=\bigcap_{g \in F} g A g^{-1}$, the group $F / A_{0}$ is the group of the group extension $\tilde{\pi}$ associated with the equicontinuous extension $\pi$. More precisely, there exists a minimal dynamical system $(\tilde{X}, \Gamma)$, with $\mathfrak{G}\left(\tilde{X}, \tilde{x}_{0}\right)=A_{0}$, on which the compact Hausdorff topological group $K=F / A_{0}$ acts as a group of automorphisms and we have the following commutative diagram

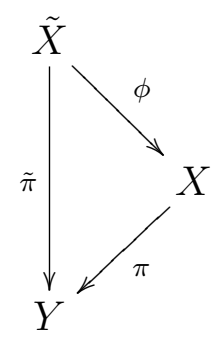

where $\tilde{\pi}: \tilde{X} \rightarrow Y \cong \tilde{X} / K$ is a group extension and so is the extension $\phi: \tilde{X} \rightarrow X \cong$ $\tilde{X} / L$ with $L=A / A_{0} \subset F / A_{0}=K$. ( $\tilde{X}=X$ iff $A$ is a normal subgroup of $F$.)

A minimal system $(X, \Gamma)$ is called incontractible if the union of minimal subsets is dense in every product system $\left(X^{n}, \Gamma\right)$. This is the case iff $p \circ G x=X$ for some (hence every) $x \in X$ and $p \in M$. When $\Gamma$ is abelian $G x$ is always dense in $X$ so that every minimal system is incontractible. However the following relative notion is an important tool even when $\Gamma$ is abelian.

We say that $(X, \Gamma) \stackrel{\pi}{\rightarrow}(Y, \Gamma)$ is a RIC (relatively incontractible) extension if:

for every $y=p y_{0} \in Y, p$ an element of $M, \pi^{-1}(y)=p \circ u \pi^{-1}\left(y_{0}\right)=p \circ F x_{0}$, where $F=\mathfrak{G}\left(Y, y_{0}\right)$.

One can show that every RIC extension is open and that every distal extension is RIC. It then follows that every distal extension is open.

We have the following theorem from [12] about the interpolation of equicontinuous extensions. For a proof see [16], Theorem X.2.1.

1.1. Theorem. Let $\pi: X \rightarrow Y$ be a RIC extension of minimal systems. Fix a point $x_{0} \in X$ with $u x_{0}=x_{0}$ and let $y_{0}=\pi\left(x_{0}\right)$. Let $A=\mathfrak{G}\left(X, x_{0}\right)$ and $F=\mathfrak{G}\left(Y, y_{0}\right)$. Then there exists a commutative diagram of pointed systems

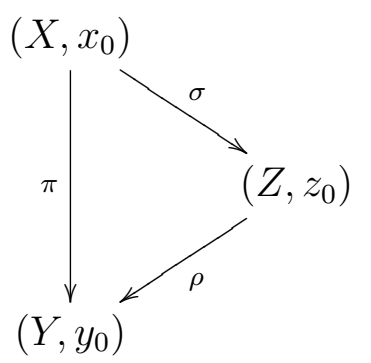

such that $\rho$ is the largest intermediate equicontinuous extension. Its Ellis group satisfies $\mathfrak{G}\left(Z, z_{0}\right)=A F^{\prime}$. The extension $\rho$ is an isomorphism iff $A F^{\prime}=F$.

Given a homomorphism $\pi:(X, \Gamma) \rightarrow(Y, \Gamma)$ of minimal metric systems, there are several standard constructions of associated "shadow diagrams". In the O-shadow 
diagram

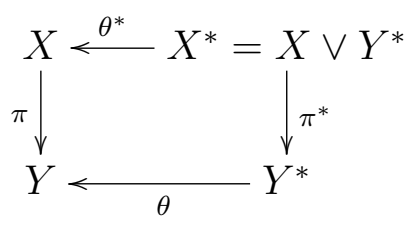

the map $\pi^{*}$ is open and the maps $\theta$ and $\theta^{*}$ are almost $1-1$. The explicit constructions is as follows. The set valued map $\pi^{-1}: Y \rightarrow 2^{X}$ (where the latter is the compact space of closed subsets of $X$, equipped with the Hausdorff, or Vietoris, topology) is upper-semicontinuous and we let $Y_{0} \subset Y$ be the set of continuity points of this map. Set $Y^{*}=\operatorname{cls}\left\{\pi^{-1}(y): y \in Y_{0}\right\} \subset 2^{X}$, and $X^{*}=X \vee Y^{*}=\operatorname{cls}\left\{\left(x, \pi^{-1}(y)\right): y \in\right.$ $\left.Y_{0}, \pi(x)=y\right\} \subset X \times Y^{*}$. By the upper-semicontinuity of $\pi^{-1}$ every $y^{*} \in Y^{*}$ is contained in a fiber $\pi^{-1}(y)$ for some $y \in Y$ and we let $\theta\left(y^{*}\right)=y$. The maps $\pi^{*}$ and $\theta^{*}$ are the restriction to $X^{*}$ of the coordinate projections on $X$ and $Y^{*}$ respectively. One then shows that $X^{*}=\left\{\left(x, y^{*}\right): x \in y^{*} \in Y^{*}\right\}$ and that indeed, $\pi^{*}$ is open and the maps $\theta$ and $\theta^{*}$ are highly proximal. The O-shadow diagram collapses, i.e. $Y=Y^{*}$, $X=X^{*}$ and $\pi=\pi^{*}$ iff $\pi: X \rightarrow Y$ is an open map; iff the map $\pi^{-1}: Y \rightarrow 2^{X}$ is continuous.

In the $R I C$-shadow diagram

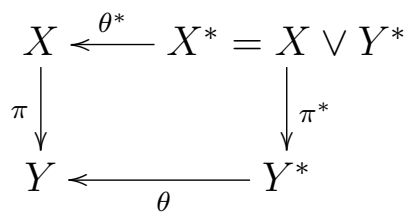

$\pi^{*}$ is RIC and $\theta, \theta^{*}$ are proximal (thus we still have $A=\mathfrak{G}\left(X, x_{0}\right)=\mathfrak{G}\left(X^{*}, x_{0}^{*}\right)$ and $\left.F=\mathfrak{G}\left(Y, y_{0}\right)=\mathfrak{G}\left(Y^{*}, y_{0}^{*}\right)\right)$. The concrete description of these objects uses quasifactors and the circle operation:

$$
Y^{*}=\left\{p \circ F x_{0}: p \in M\right\} \subset 2^{X}, \quad X^{*}=\left\{\left(x, y^{*}\right): x \in y^{*} \in Y^{*}\right\} \subset X \times Y^{*}
$$

and

$$
\theta\left(p \circ F x_{0}\right)=p y_{0}, \quad \theta^{*}\left(x, y^{*}\right)=x, \quad \pi^{*}\left(x, y^{*}\right)=y^{*}, \quad(p \in M),
$$

where $F=\mathfrak{G}\left(Y, y_{0}\right)$. The map $\theta$ is an isomorphisms (hence $\pi=\pi^{*}$ ) when and only when $\pi$ is already RIC.

We say that $\pi:(X, \Gamma) \rightarrow(Y, \Gamma)$ has a relatively invariant measure (or that is a RIM extension), if there exists a projection $P: C(X) \rightarrow C(Y)$ such that

1. $P(f) \geq 0$ for $f \geq 0$ in $C(X)$.

2. $P(\mathbf{1})=1$.

3. $P(h \circ \pi)=h$ for every $h \in C(Y)$.

4. $P(f \circ \gamma)=P(f) \circ \gamma$ for every $f \in C(X)$ and $\gamma \in \Gamma$.

This property is equivalent to the existence of a continuous section, i.e. a continuous $\Gamma$ equivariant map $y \mapsto \lambda_{y}$ from $Y$ into $\mathfrak{M}(X)$ such that $\pi\left(\lambda_{y}\right)=\delta_{y}$ for every $y \in$ $Y$. Here and in the sequel we use the same letter $\pi$ to denote the induced map $\pi: \mathfrak{M}(X) \rightarrow \mathfrak{M}(Y)$ on the spaces of probability measures. Sometimes though we will write $\pi_{*}$ for the induced map.

In the sequel we will need the following lemmas. 
1.2. Lemma. Let $\pi:(X, \Gamma) \rightarrow(Y, \Gamma)$ be a RIM homomorphism of minimal metric systems with section $y \mapsto \lambda_{y}$. Then there exists a dense $G_{\delta}$ subset $Y_{f s} \subset Y$ with the property that $\operatorname{supp}\left(\lambda_{y}\right)=\pi^{-1}(y)$ for every $y \in Y_{f s}$.

Proof. It is easy to see that, in general, the map $y \mapsto \operatorname{supp}\left(\lambda_{y}\right)$ from $Y$ into $2^{X}$ is lower-semicontinuous; i.e. whenever $y_{i} \rightarrow y$ in $Y$ then $\liminf \operatorname{supp}\left(\lambda_{y_{i}}\right) \supset \operatorname{supp}\left(\lambda_{y}\right)$. Such a map admits a dense $G_{\delta}$ set of continuity points which we denote by $Y_{f s}$. We claim that for each $y \in Y_{f s}$ the measure $\lambda_{y}$ has full support, i.e. $\operatorname{supp}\left(\lambda_{y}\right)=\pi^{-1}(y)$. To see this observe first that, by minimality, cls $\left(\cup\left\{\operatorname{supp}\left(\lambda_{y}\right): y \in Y\right\}\right)=X$. Now if $y \in Y_{f s}$ and $x \in \pi^{-1}(y)$ then there is a sequence $x_{i} \rightarrow x$ with $x_{i} \in \operatorname{supp}\left(\lambda_{y_{i}}\right)$, for some sequence $y_{i} \in Y$. Necessarily $\pi\left(x_{i}\right)=y_{i} \rightarrow y$ and, as $y \in Y_{f s}$, we have $\lim \operatorname{supp}\left(\lambda_{y_{i}}\right)=\operatorname{supp}\left(\lambda_{y}\right)$. Now

$$
x \in \liminf \operatorname{supp}\left(\lambda_{y_{i}}\right)=\lim \operatorname{supp}\left(\lambda_{y_{i}}\right)=\operatorname{supp}\left(\lambda_{y}\right) \text {. }
$$

1.3. Lemma. Let $\pi:(X, \Gamma) \rightarrow(Y, \Gamma)$ be a RIM homomorphism of minimal metric systems with section $y \mapsto \lambda_{y}$. Let (1.4) be the associated O-shadow diagram. Then the open homomorphism $\pi^{*}$ is RIM as well.

Proof. We use the notations following the definition of the O-shadow diagram (1.4). Let $Y_{0} \ni y_{i} \rightarrow y \in Y \backslash Y_{0}$ and let $Y^{*} \ni y^{*}=\lim \pi^{-1}\left(y_{i}\right)$. By the lower-semicontinuity of the map $\lambda_{y} \mapsto \operatorname{supp}\left(\lambda_{y}\right)$ we see that $\operatorname{supp}\left(\lambda_{y}\right) \subset y^{*}$. Thus for every such $y$ $\operatorname{supp}\left(\lambda_{y}\right) \subset \bigcap\left\{y^{*} \in Y^{*}: y^{*} \subset \pi^{-1}(y)\right\}$. It is now easy to check that the map $y^{*} \mapsto \lambda_{\theta\left(y^{*}\right)} \times \delta_{y^{*}}$ is a section for $\pi^{*}$.

Given a homomorphism $\pi:(X, \Gamma) \rightarrow(Y, \Gamma)$ of minimal metric systems there is an associated RIM shadow diagram

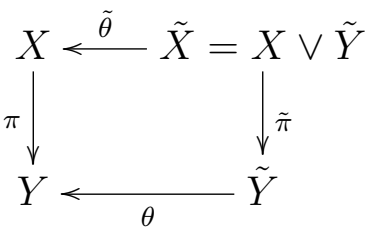

the map $\tilde{\pi}$ has a RIM and the maps $\theta$ and $\tilde{\theta}$ are strongly proximal. It can be shown that every isometric extension has a RIM and is open. See [15] for more details, also a treatment of SPI systems can be found in [17].

We say that a minimal system $(X, \Gamma)$ is a strictly $P I$ system if there is an ordinal $\eta$ (which is countable when $X$ is metrizable) and a family of systems $\left\{\left(W_{\iota}, w_{\iota}\right)\right\}_{\iota \leq \eta}$ such that (i) $W_{0}$ is the trivial system, (ii) for every $\iota<\eta$ there exists a homomorphism $\phi_{\iota}: W_{\iota+1} \rightarrow W_{\iota}$ which is either proximal or equicontinuous (isometric when $X$ is metrizable), (iii) for a limit ordinal $\nu \leq \eta$ the system $W_{\nu}$ is the inverse limit of the systems $\left\{W_{\iota}\right\}_{\iota<\nu}$, and (iv) $W_{\eta}=X$. We say that $(X, \Gamma)$ is a PI-system if there exists a strictly PI system $\tilde{X}$ and a proximal homomorphism $\theta: \tilde{X} \rightarrow X$.

If in the definition of (strictly) PI-systems we replace proximal extensions by HP extensions (almost 1-1 extensions in the metric case) we get the notion of (strictly) HPI (AI-systems in the metric case). If we replace the proximal extensions by trivial extensions (i.e. we do not allow proximal extensions at all) we have I-systems. In this 
terminology the structure theorem for distal systems (Furstenberg [13], 1963) can be stated as follows:

1.4. Theorem. A metric minimal system is distal iff it is an I-system.

And, the Veech-Ellis structure theorem for point distal systems (Veech [33], 1970 and Ellis [8], 1973):

1.5. Theorem. A metric minimal dynamical system is point distal iff it is an AIsystem.

The structure theorem for the general minimal system is proved in [12] and [31] (see also [33]) and asserts that every minimal system admits a canonically defined proximal extension which is a weakly mixing RIC extension of a strictly PI system. Both the Furstenberg and the Veech-Ellis structure theorems are corollaries of this general structure theorem.

1.6. Theorem (Structure theorem for minimal systems). Given a minimal system $(X, \Gamma)$, there exists an ordinal $\eta$ (countable when $X$ is metrizable) and a canonically defined commutative diagram (the canonical PI-Tower)

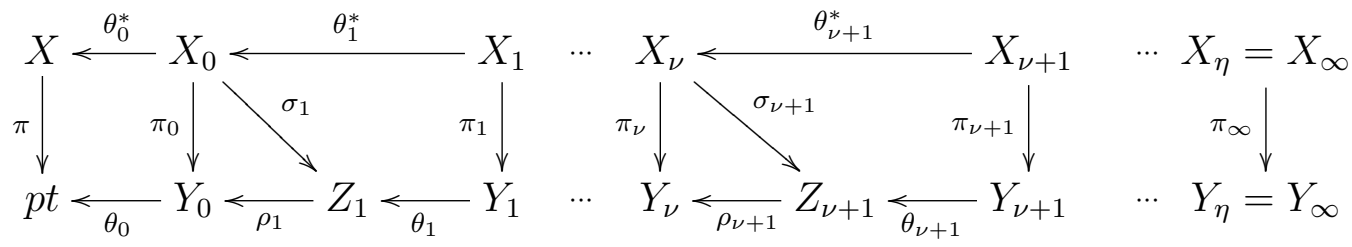

where for each $\nu \leq \eta, \pi_{\nu}$ is $R I C, \rho_{\nu}$ is isometric, $\theta_{\nu}, \theta_{\nu}^{*}$ are proximal and $\pi_{\infty}$ is $R I C$ and weakly mixing. For a limit ordinal $\nu, X_{\nu}, Y_{\nu}, \pi_{\nu}$ etc. are the inverse limits (or joins) of $X_{\iota}, Y_{\iota}, \pi_{\iota}$ etc. for $\iota<\nu$. Thus $X_{\infty}$ is a proximal extension of $X$ and a $R I C$ weakly mixing extension of the strictly PI-system $Y_{\infty}$. The homomorphism $\pi_{\infty}$ is an isomorphism (so that $X_{\infty}=Y_{\infty}$ ) iff $X$ is a PI-system.

Two useful criteria on minimal systems for being PI and HPI are given in theorems of Bronstein [5] and van der Woude [35], respectively.

In the sequel we will mostly deal with metrizable systems. So from now on, unless we explicitly say otherwise all the dynamical systems will be assumed to be metrizable.

All of the above mentioned theorems have their relative versions. One starts with a homomorphism $\pi:(X, \Gamma) \rightarrow(Y, \Gamma)$ between minimal systems and then all the various notions defined above, equicontinuity, distality, point-distality etc. have natural relative analogues.

We will use two such relative theorems which I now proceed to describe.

1.7. Definition. Let $\pi: X \rightarrow Y$ be a homomorphism of minimal systems. Then $\pi$ is a point distal extension if there is a point $x_{0} \in X$ such that $P\left[x_{0}\right] \cap \pi^{-1}\left(y_{0}\right)=\left\{x_{0}\right\}$, where $y_{0}=\pi\left(x_{0}\right)$. A point such as $x_{0}$ is called a $Y$-distal point.

1.8. Theorem (Veech [32], Ellis [11]). Let $\pi: X \rightarrow Y$ be a homomorphism of metric minimal systems. Then $\pi$ is point distal iff $\pi$ is an AI extension and the set of $Y$ distal points is a dense $G_{\delta}$ subset of $X$. If $\pi$ is open then it is point distal iff it is strictly $A I$. 
A homomorphism $\pi:(X, \Gamma) \rightarrow(Y, \Gamma)$ is called semiopen if the interior of $\pi(U)$ is nonempty for every nonempty open subset $U$ of $X$. When $X$ is minimal every $\pi:(X, \Gamma) \rightarrow(Y, \Gamma)$ is semiopen (see Lemma 2.5 below). We will say that a subset $W \subset X \times X$ is a $S$-set if it is closed, invariant, topologically transitive, and the restriction to $W$ of the projection maps are semiopen.

1.9. Theorem (van der Woude, [35]). A homomorphism $\pi: X \rightarrow Y$ between metric minimal systems $(X, \Gamma)$ and $(Y, \Gamma)$ is a point distal extension iff every $S$-set of $R_{\pi}=$ $\left\{\left(x, x^{\prime}\right) \in X \times X: \pi(x)=\pi\left(x^{\prime}\right)\right\}$ is minimal.

\section{ON SEMIOPEN MAPS}

2.1. Lemma. Let $\pi: X \rightarrow Y$ be a continuous surjection between compact Hausdorff spaces. The conditions 1 and 2 below are equivalent. If $X$ is metrizable then the three conditions are equivalent:

1. $\pi$ is semiopen.

2. The preimage of every dense subset in $Y$ is dense in $X$.

3. The set

$X_{0}=\left\{x \in X:\right.$ the set valued map $\pi^{-1}: Y \rightarrow 2^{X}$ is continuous at $\left.\pi(x)\right\}$ is dense in $X$.

Proof. The equivalence of 1 and 2 is straightforward. For any continuous surjection $\pi: X \rightarrow Y$ the corresponding set map $\pi^{-1}: Y \rightarrow 2^{X}$ is upper-semicontinuous and, when $X$ is metrizable, this implies that it has a dense $G_{\delta}$ subset $Y_{0} \subset Y$ of continuity points. Assuming 2 we conclude that $X_{0}=\pi^{-1}\left(Y_{0}\right)$ is a dense $G_{\delta}$ subset of $X$. Conversely if 3 is valid and $U \subset X$ is open and nonempty, then $U \cap X_{0} \neq \emptyset$ and if $x_{0}$ is any point in this intersection then $\pi$ is open at $x_{0}$, so that $\pi(U)$ is a neighborhood of $\pi\left(x_{0}\right)$ and we conclude that $\pi(U)^{\circ} \neq \emptyset$.

2.2. Lemma. Let $X \stackrel{\alpha}{\rightarrow} Z \stackrel{\beta}{\rightarrow} Y$, where $X, Y$ and $Z$ are compact metric spaces, $\alpha$ and $\beta$ are continuous surjections, and $\alpha$ is semiopen. For $y \in Y$ set $Z_{y}=\beta^{-1}(y)$ and $X_{y}=(\beta \circ \alpha)^{-1}(y)=\alpha^{-1}\left(Z_{y}\right)$. Then there is a dense $G_{\delta}$ subset $Y_{0} \subset Y$ such that for every $y \in Y_{0}$ the restriction $\alpha: X_{y} \rightarrow Z_{y}$ is semiopen.

Proof. Let $V \subset X$ be a nonempty open subset. Set

$$
Y_{V}=\left\{y \in Y: \alpha(V)^{\circ} \cap Z_{y} \neq \emptyset\right\} \cup\left\{y \in Y: \bar{V} \cap X_{y}=\emptyset\right\}:=Y_{V, 1} \cup Y_{V, 2} .
$$

We will show that $V_{Y}$ is open and dense.

Suppose $y_{n} \rightarrow y$ is a convergent sequence with $y_{n} \notin Y_{V}$. Then, as $Y_{V}^{c}=Y_{V, 1}^{c} \cap Y_{V, 2}^{c}$, we have

- For all $n$

$$
\begin{gathered}
y_{n} \in Y_{V, 1}^{c} \Rightarrow \alpha(V)^{\circ} \cap Z_{y_{n}}=\emptyset \Rightarrow Z_{y_{n}} \subset\left(\alpha(V)^{\circ}\right)^{c} \Rightarrow \\
Z_{y} \subset\left(\alpha(V)^{\circ}\right)^{c} \Rightarrow \alpha(V)^{\circ} \cap Z_{y}=\emptyset \Rightarrow y \in Y_{V, 1}^{c} .
\end{gathered}
$$

- And, for all $n$

$$
\begin{gathered}
y_{n} \in Y_{V, 2}^{c} \Rightarrow \bar{V} \cap X_{y_{n}} \neq \emptyset \Rightarrow \bar{V} \cap \limsup X_{y_{n}} \neq \emptyset \Rightarrow \\
\bar{V} \cap X_{y} \neq \emptyset \Rightarrow y \in Y_{V, 2}^{c} .
\end{gathered}
$$


This shows that $Y_{V}$ is open.

Next let $O \subset Y$ be a nonempty open set. We will show that $O \cap Y_{V} \neq \emptyset$.

- Case1 : Suppose $\beta^{-1}(O) \cap \overline{\alpha(V)} \neq \emptyset$.

Then $\beta^{-1}(O) \cap\left(\alpha(V)^{\circ} \cup \partial(\alpha(V)) \neq \emptyset\right.$. As $\alpha$ is a semiopen map, the set $\partial(\alpha(V))$ has an empty interior and it follows that $\beta^{-1}(O) \cap \alpha(V)^{\circ} \neq \emptyset$. Let $z$ be a point in this intersection and set $y=\beta(z)$. Then $y \in O$ and $z \in \alpha(V)^{\circ} \cap Z_{y}$ imply that $O \cap Y_{V, 1} \neq \emptyset$.

- Case 2 : Suppose $\beta^{-1}(O) \cap \overline{\alpha(V)}=\emptyset$.

It then follows that for every $y \in O$ we have $\bar{V} \cap X_{y}=\emptyset$, whence $y \in O \cap Y_{V, 2}$.

Let now $\left\{V_{i}\right\}_{\in \mathbb{N}}$ be an enumeration of a basis for open sets on $X$. Set $Y_{i}=Y_{V_{i}}$ and let $Y_{0}=\cap_{i \in \mathbb{N}} Y_{i}$.

We now check that the set $Y_{0} \subset Y$ satisfies the requirement of the lemma. In fact, suppose $y \in Y_{0}$. We want to show that $\alpha: X_{y} \rightarrow Z_{y}$ is semiopen. Let then $U \subset X_{y}$ be a nonempty open subset. With no loss of generality we assume that $U=V \cap X_{y}$ for some $V$ in our basis $\left\{V_{i}\right\}_{i \in \mathbb{N}}$. In particular then $V \cap X_{y} \neq \emptyset$, whence $y \notin Y_{V, 2}$. But $y \in Y_{0} \subset Y_{V}$, hence $y \in Y_{V, 1}$, i.e. $\alpha(V)^{\circ} \cap Z_{y} \neq \emptyset$. If $z$ is a point in this intersection then $z=\alpha(x)$ for some $x \in V \cap X_{y}=U$. Thus $\alpha(U)^{\circ} \neq \emptyset$ and our proof is complete.

2.3. Corollary. Let $\pi: X \rightarrow Y$ be a continuous semiopen surjection between compact metric spaces. Let $R_{\pi}=\left\{\left(x, x^{\prime}\right) \in X \times X: \pi(x)=\pi\left(x^{\prime}\right)\right\}$. Suppose $W \subset R_{\pi}$ is a closed subset such that $(\pi \times \pi)(W)=\Delta_{Y}=\{(y, y): y \in Y\}$. Suppose further that the restrictions of the projection maps $\mathbf{p}_{i}: X \times X \rightarrow X(i=1,2)$ to $W$ are semiopen. Then there is a dense $G_{\delta}$ subset $Y_{\text {so }} \subset Y$ such that for each $y \in Y_{\text {so }}$ the maps $\mathbf{p}_{i}: W \cap\left(\pi^{-1}(y) \times \pi^{-1}(y)\right) \rightarrow \pi^{-1}(y)$ are semiopen.

Proof. Apply Lemma 2.2 to $W \stackrel{\mathbf{p}_{3}}{\rightarrow} X \stackrel{\pi}{\rightarrow} Y$ with $i=1,2$ and then take let $Y_{\text {so }}$ be the intersection of the two corresponding dense $G_{\delta}$ sets of $Y$.

A result of Ditor and Eifler from 1972, [6] asserts that a continuous surjection $\pi: X \rightarrow Y$ between compact Hausdorff spaces $X$ and $Y$ is open iff the induced map $\pi_{*}: \mathfrak{M}(X) \rightarrow \mathfrak{M}(Y)$ is an open surjection. In the course of the proof of our main theorem (Theorem 5.3) we will need the following analogous result (in the metric case) for semiopen maps. For the proof we refer to [21, Theorem 2.3].

2.4. Theorem. Let $\pi: X \rightarrow Y$ be a continuous surjection between compact metric spaces which is semiopen. Then the induced map $\pi_{*}: \mathfrak{M}(X) \rightarrow \mathfrak{M}(Y)$ is a semiopen surjection.

Recall the following well known result; for completeness we include a proof.

2.5. Lemma. Let $\pi:(X, \Gamma) \rightarrow(Y, \Gamma)$ be a homomorphism between minimal systems. Then $\pi$ is semiopen.

Proof. Let $W \subset X$ be a closed set with nonempty interior. By minimality of $(X, \Gamma)$ there is a finite set $\left\{\gamma_{1}, \ldots, \gamma_{n}\right\} \subset \Gamma$ with $X=\bigcup_{i=1}^{n} \gamma_{i} W$. Therefore $Y=$ $\bigcup_{i=1}^{n} \pi\left(\gamma_{i} W\right)$ and it follows that for some $i$ the interior of the closed set $\pi\left(\gamma_{i} W\right)=$ $\gamma_{i} \pi(W)$ is nonempty. Thus, as required, also $\pi(W)^{\circ} \neq \emptyset$. 


\section{A KEY PROPOSITION ON DIFFUSED MEASURES}

As explained above we fix a minimal ideal $M$ in $\beta \Gamma$ and let $u$ be an idempotent in $M$. We denote the subgroup $u M$ of $M$ by $G$ and identify it with the group of automorphisms of the universal $\Gamma$-minimal system $(M, \Gamma)$, where for $\alpha \in G$ the corresponding automorphism $R_{\alpha}: M \rightarrow M$ is given by right multiplication $p \mapsto p \alpha$. For an abelian $\Gamma$ each subgroup $v M \subset M$, where $v$ is an idempotent in $M$, is dense in $M$ and it follows that the $G$-dynamical system $(M, G)$ (where $G$ acts by right multiplication) is minimal. In the general case the compact dynamical system $(M, G)$ admits a minimal subset and it follows that there is a minimal idempotent $v$ such that $\operatorname{cls}(v G)$ is minimal under the right $G$ action. In the sequel we will need the following slightly stronger statement.

\subsection{Lemma. If $(Y, \Gamma)$ is a minimal proximal system then}

1. For every minimal idempotent $v \in M$ there is a unique point $y_{0} \in Y$ with $v y_{0}=y_{0}$ and moreover, $v y=y_{0}$ for every $y \in Y$.

2. For every $y \in Y$ there is a minimal idempotent $v \in M$ such that $v y=y$ and the G-system (cls $(v G), G)$ is minimal.

Proof. The first assertion is clear. For the second observe that the closed set $\{p \in M$ : $p y=y\}$ is $G$-invariant (under the right action of $G$ ) and hence contains a $G$-minimal set. Clearly this set contains an idempotent, and any such idempotent $v$ satisfies the required property.

As our choice of $u$ was arbitrary we can, and will from now on, assume that $\operatorname{cls}(u G)=\operatorname{cls}(G u)=\operatorname{cls}(G)$ is $G$-minimal.

3.2. Lemma. Let $\left(Z^{*}, \Gamma\right)$ be a minimal metric system and let $\phi:\left(Z^{*}, \Gamma\right) \rightarrow(Y, \Gamma)$ be its maximal proximal factor. Suppose further that we have the following diagram

$$
Z^{*} \stackrel{\theta}{\rightarrow} Z \stackrel{\sigma}{\rightarrow} Y
$$

where $\sigma$ is a maximal isometric (i.e. equicontinuous) extension, $\theta$ is an almost 1-1 extension, and $\phi=\theta \circ \sigma$ (i.e. $\phi$ is an almost automorphic extension). Let $O$ be a nonempty open subset of $Z^{*}$.

1. There is a nonempty open subset $V \subset O$ such that $\theta^{-1}(\theta(V)) \subset O$.

2. There is a nonempty open subset $W \subset O$ such that $\operatorname{cls}_{\tau}\left(W \cap u Z^{*}\right) \subset O$.

Proof. 1. Suppose $O \subset Z^{*}$ is a nonempty open set for which the statement of the lemma fails. Choose a point $z^{*} \in O$ such that $\theta^{-1}\left(\theta\left(z^{*}\right)\right)=\left\{z^{*}\right\}$ and let $V_{n} \subset O$ be a sequence of open balls centered at $z^{*}$ with $\operatorname{diam}\left(V_{n}\right) \searrow 0$. By assumption there are pairs of points $z_{n} \in V_{n}$ and $z_{n}^{\prime} \notin O$ with $\theta\left(z_{n}\right)=\theta\left(z_{n}^{\prime}\right)$. However, as $\lim _{n \rightarrow \infty} \theta^{-1}\left(\theta\left(z_{n}\right)\right)=\left\{z^{*}\right\}$, we have $z_{n}^{\prime} \rightarrow z^{*}$ in contradiction of the fact that $O$ is a neighborhood of $z^{*}$.

2. Since $Z$ is an isometric extension of the proximal system $Y, u Z$ is a closed subset of $Z$ and the $\tau$-topology on $u Z$ coincides with its compact Hausdorff group topology. Since $\theta$ is an almost one-to-one map, the restriction $\theta \uparrow u Z^{*}: u Z^{*} \rightarrow u Z$ is a homeomorphism of $u Z^{*}$, equipped with the $\tau$ topology, onto $u Z$. Let $O \subset Z^{*}$ be a nonempty open set. Let $V \subset O$ be as in part 1 , and let $W$ be a nonempty open 
subset such that $\bar{W} \subset V$. Now

$$
\begin{aligned}
\operatorname{cls}_{\tau}\left(W \cap u Z^{*}\right) & =\theta^{-1}\left(\operatorname{cls}_{\tau}(\theta(W \cap u Z)) \cap u Z^{*}\right. \\
& =\theta^{-1}(\overline{\theta(W \cap u Z)}) \cap u Z^{*} \\
& \subset \theta^{-1}(\overline{\theta(W)})=\theta^{-1}(\theta(\bar{W})) \\
& \subset \theta^{-1}(\theta(V)) \subset O .
\end{aligned}
$$

3.3. Lemma. Let $(X, \Gamma)$ be a minimal metric system and let $\phi:(X, \Gamma) \rightarrow(Y, \Gamma)$ be its maximal proximal factor. Suppose further that we have the following diagram

$$
X \stackrel{\rho}{\rightarrow} Z^{*} \stackrel{\theta}{\rightarrow} Z \stackrel{\sigma}{\rightarrow} Y
$$

where $\rho$ and $\sigma$ are maximal isometric extensions, $\theta$ is an almost 1 - 1 extension, and $\phi=\sigma \circ \theta \circ \rho$. Fix a minimal ideal $M \subset \beta \Gamma$ and an idempotent $u \in M$ as above. Let $U$ be an open subset of $X$ such that $U \cap u X \neq \emptyset$. Then

$$
\operatorname{cls}_{\tau}(U \cap u X) \supset \rho^{-1}(\rho(U)) \cap u X .
$$

Proof. Fix a point $x_{0} \in X$ with $u x_{0}=x_{0}$ and let $z_{0}^{*}, z_{0}$ and $y_{0}$ be its images in $Z^{*}, Z$ and $Y$ respectively. Let

$$
\begin{gathered}
A=\mathfrak{G}\left(X, x_{0}\right)=\left\{\alpha \in G: \alpha x_{0}=x_{0}\right\}, \text { and } \\
F=\mathfrak{G}\left(Z, z_{0}\right)=\mathfrak{G}\left(Z^{*}, z_{0}^{*}\right)=\left\{\alpha \in G: \alpha z_{0}=z_{0}\right\}=\left\{\alpha \in G: \alpha z_{0}^{*}=z_{0}^{*}\right\} .
\end{gathered}
$$

Note that, as $Y$ is a proximal system, we have $\mathfrak{G}\left(Y, y_{0}\right)=G$. The assumption that $\rho$ is an isometric extension implies that $B=F / A$ is a homogeneous space of the Hausdorff compact topological group $F / \cap\left\{f A f^{-1}: f \in F\right\}$ (with respect to its $\tau$-topology). The fact that $(Z, \Gamma)$ is the maximal equicontinuous extension of $(Y, \Gamma)$ (within $X$ ) implies that $F \supset G^{\prime}$ and that $G^{\prime} A=A G^{\prime}=F$. Let $h: M \rightarrow X$ denote the evaluation map $p \mapsto p x_{0}$.

Let $U$ be a nonempty open subset of $X$ such that $U \cap u X \neq \emptyset$. Set $\tilde{U}=h^{-1}(U)=$ $\left\{p \in M: p x_{0} \in U\right\}$. Then $\tilde{U}$ is an open subset of $M$ with $\tilde{U} \cap G \neq \emptyset$ and, by minimality of the $G$-system $(\operatorname{cls}(G), G)$, the collection $\{\tilde{U} \alpha: \alpha \in G\}$ is an open cover of $\operatorname{cls} G$. Choose a finite subcover, say $\left\{\tilde{U} \alpha_{i}: i=1,2, \ldots, n\right\}$. Now

$$
\bigcup_{i=1}^{n} \operatorname{cls}_{\tau}\left(\tilde{U} \alpha_{i} \cap G\right)=\bigcup_{i=1}^{n} \operatorname{cls}_{\tau}(\tilde{U} \cap G) \alpha_{i}=G ;
$$

hence $\operatorname{cls}_{\tau}(\tilde{U} \cap G)$ has a nonempty $\tau$-interior. Since $\operatorname{cls}_{\tau}(\tilde{U} \cap G)$ is also $\tau$-closed, it must contain a left translate of $G^{\prime}$, say $\beta G^{\prime}$ for some $\beta \in G$ (this follows from the definition of $G^{\prime}$, see equation (1.1)). Projecting back to $X$ via $h$ we get

$$
\begin{aligned}
\operatorname{cls}_{\tau}(U \cap u X) & =h\left(\operatorname{cls}_{\tau}(\tilde{U} \cap G)\right) \\
& \supset \beta G^{\prime} x_{0}=\beta G^{\prime} A x_{0}=\beta F x_{0} \\
& =\left(\beta F \beta^{-1}\right) \beta x_{0}=\rho^{-1}\left(\rho\left(\beta x_{0}\right)\right) .
\end{aligned}
$$

Let $O=\rho(U)$, then $O$ is a nonempty open subset of $Z^{*}$ and by Lemma 3.2 there is a nonempty open subset $W \subset O$ such that $\operatorname{cls}_{\tau}\left(W \cap u Z^{*}\right) \subset O$. Set $U_{1}=\rho^{-1}(W) \cap U$. 
Then $U_{1}$ is a nonempty open subset of $X$ and by the above argument there exists $\beta_{1} \in G$ with $\rho^{-1}\left(\rho\left(\beta_{1} x_{0}\right)\right) \subset \operatorname{cls}_{\tau}\left(U_{1} \cap u X\right) \subset \operatorname{cls}_{\tau}(U \cap u X)$. Now

$$
\begin{aligned}
\rho\left(\beta_{1} x_{0}\right) & \in \rho\left(\operatorname{cls}_{\tau}\left(U_{1} \cap u X\right)\right) \\
& =\operatorname{cls}_{\tau}\left(\rho\left(U_{1}\right) \cap u Z^{*}\right) \\
& \subset \operatorname{cls}_{\tau}\left(W \cap u Z^{*}\right) \subset O=\rho(U) .
\end{aligned}
$$

Thus we have shown that for every nonempty open subset $U \subset X$ which meets $u X$, the set $\operatorname{cls}_{\tau}(U \cap u X)$ contains a full fiber $\rho^{-1}\left(z^{*}\right)$ for some $z^{*} \in \rho(U)$. Since $\rho$ is an open map we conclude that

$$
\operatorname{cls}_{\tau}(U \cap u X) \supset \rho^{-1}(\rho(U)) \cap u X
$$

as required.

This lemma has the following surprizing corollary.

3.4. Corollary. In the situation described in Lemma 3.3, if $\sigma: Z \rightarrow Y$ is a finite to one isometric extension then $\rho: X \rightarrow Z^{*}$ is an isomorphism, i.e. $\phi: X \rightarrow Y$ (with $\phi=\sigma \circ \theta \circ \rho)$ is an almost automorphic extension. In particular if, in addition, $Y$ is the trivial one point system, then $X=Z$ is a finite system.

Proof. Let $z_{0} \in Z$ be a point with $\theta^{-1}\left(z_{0}\right)=\left\{z_{0}^{*}\right\}$ a singleton. With no loss of generality we can assume that $u z_{0}=z_{0}$ (see Lemma 3.1). Note that then also $u z_{0}^{*}=z_{0}^{*}$ and $\rho^{-1}\left(z_{0}^{*}\right) \subset u X$. Let $y_{0}=u y_{0}=\sigma\left(z_{0}\right) \in Y$ and let $X_{0}=\phi^{-1}\left(y_{0}\right)$, $Z_{0}^{*}=\rho\left(X_{0}\right)$ and $Z_{0}=\sigma^{-1}\left(y_{0}\right)=u Z$. By our assumptions $Z_{0}$ is a finite set and therefore $\left\{z_{0}^{*}\right\}$ is a relatively open subset of $\mathrm{u} Z_{0}^{*}$. Then $\rho^{-1}\left(z_{0}^{*}\right)$ is a relatively open subset of $X_{0}$. We claim that $\rho^{-1}\left(z_{0}^{*}\right)$ is a singleton. If this is not the case, then there is an open subset $U \subset X$ with $\emptyset \neq \bar{U} \cap \rho^{-1}\left(z_{0}^{*}\right) \subsetneq \rho^{-1}\left(z_{0}^{*}\right)$. By Lemma 3.3 we have

$$
\operatorname{cls}_{\tau}(U \cap u X) \supset \rho^{-1}(\rho(U)) \cap u X .
$$

But $\operatorname{cls}_{\tau}(U \cap u X) \subset \bar{U}$ (because $\rho$ is an isometric extension) and $z_{0}^{*} \in \rho(U)$, whence $\rho^{-1}(\rho(U)) \cap u X \supset \rho^{-1}\left(z_{0}^{*}\right)$. It follows that the set $\bar{U}$ contains the whole fiber $\rho^{-1}\left(z_{0}^{*}\right)$, a contradiction. Thus $\rho^{-1}\left(z_{0}^{*}\right)$ is indeed a singleton and it follows that $\rho$ is one-to-one as claimed.

3.5. Proposition. Let $(X, \Gamma)$ be a minimal metric system and let $\phi:(X, \Gamma) \rightarrow(Y, \Gamma)$ be its maximal proximal factor. Suppose further that we have the following diagram

$$
X \stackrel{\rho}{\rightarrow} Z^{*} \stackrel{\theta}{\rightarrow} Z \stackrel{\sigma}{\rightarrow} Y,
$$

where $\rho$ and $\sigma$ are maximal isometric extensions, $\theta$ is an almost 1 -1 extension, and $\phi=\sigma \circ \theta \circ \rho$.

1. For every $z^{*} \in Z^{*}$ the fiber $\rho^{-1}\left(z^{*}\right)$ has the structure of a homogeneous space of a compact Hausdorff topological group and we let $\lambda_{z^{*}}$ be the corresponding Haar measure on this fiber. In particular $\rho$ is a RIM and open extension and $z^{*} \mapsto \lambda_{z^{*}}, Z^{*} \rightarrow \mathfrak{M}(X)$, is the corresponding section. Let $\Lambda: \mathfrak{M}\left(Z^{*}\right) \rightarrow \mathfrak{M}(X)$, defined by

$$
\Lambda(\nu)=\int_{Z^{*}} \lambda_{z^{*}} d \nu\left(z^{*}\right),
$$


be the associated affine injection.

2. Let $y_{0} \in Y$ be the unique point with uy $y_{0}=y_{0}$. Denote $Z_{0}=\sigma^{-1}\left(y_{0}\right), X_{0}=$ $\phi^{-1}\left(y_{0}\right)$ and $Z_{0}^{*}=(\sigma \circ \theta)^{-1}\left(y_{0}\right)=\rho\left(X_{0}\right)$. Set $\mathfrak{M}_{m}\left(X_{0}\right)=\left\{\Lambda(\nu): \nu \in \mathfrak{M}\left(Z_{0}^{*}\right)\right\}$. Then the set

$$
R=\left\{\nu \in \mathfrak{M}\left(X_{0}\right): \text { the orbit closure of } \nu \text { meets } \mathfrak{M}_{m}\left(X_{0}\right)\right\}
$$

is a dense $G_{\delta}$ subset of $\mathfrak{M}\left(X_{0}\right)$.

Proof. Part 1 is well known; see e.g. Corollary 3.7 in [15].

2. Fix a compatible metric $d$ on $\mathfrak{M}(X)$. Let $\kappa \in \mathfrak{M}\left(X_{0}\right)$ and $\epsilon, \eta>0$ be given. Find an atomic measure $\lambda=\frac{1}{n} \sum_{i=1}^{n} \delta_{x_{i}}, x_{i} \in X_{0}$ such that $d(\kappa, \lambda)<\epsilon / 2$. Choose open disjoint neighborhoods $U_{i}$ of $x_{i}$, so small that every measure of the form $\mu=$ $\frac{1}{n} \sum_{i=1}^{n} \mu_{i}, \mu_{i} \in \mathfrak{M}\left(X_{0}\right)$ with $\operatorname{supp} \mu_{i} \subset U_{i}$, will satisfy $d(\mu, \lambda)<\epsilon / 2$, and hence also $d(\mu, \kappa)<\epsilon$.

Set $\nu=\frac{1}{n} \sum_{i=1}^{n} \lambda_{z_{i}^{*}}$ with $z_{i}^{*}=\rho\left(x_{i}\right)$ (an element of $\mathfrak{M}_{m}\left(X_{0}\right)$ ). For each $z_{i}^{*}$ choose points $\left\{x_{i, j}^{\prime}\right\}_{j=1}^{k} \in \rho^{-1}\left(z_{i}^{*}\right)$ so that $d\left(\mu^{\prime}, \nu\right)<\eta / 2$, where

$$
\mu^{\prime}=\frac{1}{n k} \sum_{i=1}^{n} \sum_{j=1}^{k} \delta_{x_{i, j}^{\prime}} .
$$

By Lemma 3.3

$$
\begin{gathered}
u X \cap u \circ\left(u X \cap \bigcup_{i=1}^{n} U_{i}\right)=\operatorname{cls}_{\tau}\left(u X \cap \bigcup_{i=1}^{n} U_{i}\right) \\
\supset u X \cap\left(\bigcup_{i=1}^{n} \rho^{-1}\left(\rho\left(U_{i}\right)\right)\right) .
\end{gathered}
$$

Therefore there exist an element $\gamma \in \Gamma$ and for each $i$ a set $\left\{x_{i, j}\right\}_{j=1}^{k} \subset u X \cap U_{i}$, such that $d\left(\gamma x_{i, j}, x_{i, j}^{\prime}\right)$ is so small that the inequality $d\left(\gamma \mu, \mu^{\prime}\right)<\eta / 2$ is satisfied, with

$$
\mu=\frac{1}{n k} \sum_{i=1}^{n} \sum_{j=1}^{k} \delta_{x_{i, j}} .
$$

Thus $d(\gamma \mu, \nu)<\eta$. By the choice of the small sets $U_{i}$ we also have $d(\mu, \kappa)<\epsilon$ and, as $\epsilon>0$ is arbitrary, we have shown that the open set

$$
R_{\eta}=\left\{\mu \in \mathfrak{M}\left(X_{0}\right): \text { there exists } \gamma \in \Gamma \text { with } d\left(\gamma \mu, \mathfrak{M}_{m}\left(X_{0}\right)\right)<\eta\right\}
$$

is dense in $\mathfrak{M}\left(X_{0}\right)$. Clearly $R=\bigcap\left\{R_{\eta}: \eta>0\right\}$ is the required dense $G_{\delta}$ subset of $\mathfrak{M}\left(X_{0}\right)$.

\section{Some Properties of tame minimal Systems}

4.1. Theorem. ([20]) Let $(X, \Gamma)$ be a metric tame dynamical system. Let $\mathfrak{M}(X)$ denote the compact convex set of probability measures on $X$ (with the weak $k^{*}$ topology). Then each element $p \in E(X, \Gamma)$ defines an element $p_{*} \in E(\mathfrak{M}(X), \Gamma)$ and the map $p \mapsto p_{*}$ is both a dynamical system and a semigroup isomorphism of $E(X, \Gamma)$ onto $E(\mathfrak{M}(X), \Gamma)$. 
Proof. Since $E(X, \Gamma)$ is Fréchet we have for every $p \in E$ a sequence $\gamma_{i} \rightarrow p$ of elements of $\Gamma$ converging to $p$. Now for every $f \in C(X)$ and every probability measure $\nu \in \mathfrak{M}(X)$ we get, by the Riesz representation theorem and Lebesgue's dominated convergence theorem,

$$
\gamma_{i} \nu(f)=\nu\left(f \circ \gamma_{i}\right) \rightarrow \nu(f \circ p):=p_{*} \nu(f) .
$$

Since the Baire class 1 function $f \circ p$ is well defined and does not depend upon the choice of the convergent sequence $\gamma_{i} \rightarrow p$, this defines the map $p \mapsto p_{*}$ uniquely. It is easy to see that this map is an isomorphism of dynamical systems, whence also a semigroup isomorphism. Finally as $\Gamma$ is dense in both enveloping semigroups, it follows that this isomorphism is onto.

As we have seen, when $(X, \Gamma)$ is a metrizable tame system, the enveloping semigroup $E(X, \Gamma)$ is a separable Fréchet space. Therefore, each element $p \in E$ is a limit of a sequence of elements of $\Gamma, p=\lim _{n \rightarrow \infty} \gamma_{n}$. It follows that the subset $C(p)$ of continuity points of each $p \in E$ is a dense $G_{\delta}$ subset of $X$. More generally, if $A \subset X$ is any closed subset then the set $C_{A}(p)$ of continuity points of the map $p \uparrow A: A \rightarrow X$ is a dense $G_{\delta}$ subset of $A$. For an idempotent $v=v^{2} \in E$ we write $C_{v}$ for $C_{\overline{v X}}(v)$. For a special case of the next lemma see [23, Theorem 9.2]

4.2. Lemma. Let $(X, \Gamma)$ be a metrizable tame dynamical system and $\pi: X \rightarrow Y$ a RIM extension, with section $y \mapsto \nu_{y}$, such that $(Y, \Gamma)$ is a proximal system.

1. $p \nu_{y}=\nu_{p y}$ for every $p \in E(X, \Gamma)$ and every $y \in Y$. In particular, we have $\nu_{p y}(p X)=1$.

2. For every minimal idempotent $v \in E(X, \Gamma)$ there is a unique $y \in Y$ such that $v y=y$. For such $v$ we have $v X \subset \pi^{-1}(y)$, so that $\nu_{y}(v X)=1$ and $\operatorname{supp}\left(\nu_{y}\right) \subset v X \cap \pi^{-1}(y)=v \pi^{-1}(y)$.

3. For an idempotent $v$ in $E(X, \Gamma)$ we have $C_{v} \subset v X$.

4. If $X$ is minimal then for every point $y$ of the dense $G_{\delta}$ subset $Y_{f s} \subset Y$ (see Lemma 1.2) and $v$ a minimal idempotent in $E(X, \Gamma)$ with $v y=y, C_{v}$ is a dense $G_{\delta}$ subset of $\pi^{-1}(y)$, and $v X$ is a residual subset of $\pi^{-1}(y)$.

Proof. 1. As $p: X \rightarrow X$ is a Baire 1 function, the measure $p \nu_{x}$ is well defined and the equality follows from the continuity of the section.

2. Clear.

3. Given $x \in C_{v}$ choose a sequence $x_{n} \in v X$ with $\lim _{n \rightarrow \infty} x_{n}=x$. We then have $v x=\lim _{n \rightarrow \infty} v x_{n}=\lim _{n \rightarrow \infty} x_{n}=x$, hence $C_{v} \subset v X$.

4. For $v y=y \in Y_{f s}$ we have $\operatorname{supp}\left(\nu_{y}\right)=\pi^{-1}(y)$. By part 2 we have $\nu_{y}(v X)=1$, whence $v X$ is dense in $\pi^{-1}(y)$. Now $C_{v}=C_{\overline{v X}}(v)=C_{\pi^{-1}(y)}(v)$ and it follows that $C_{v}$ is a dense $G_{\delta}$ subset of $\pi^{-1}(y)$. By part $3 C_{v} \subset v X$ and it follows that $v X$ is residual in $\pi^{-1}(y)$.

4.3. Corollary. Let $(X, \Gamma)$ be a metrizable tame dynamical system admitting a $\Gamma$ invariant probability measure, and let $v$ be a minimal idempotent in $E(X, \Gamma)$. Then $C(v) \subset v X, C(v)$ is a dense $G_{\delta}$ subset of $X$, and $v X$ is residual in $X$. 
4.4. Theorem. Let $(X, \Gamma)$ be a metrizable tame dynamical system and $\pi: X \rightarrow Y$ a $R I M$ extension, with section $y \mapsto \nu_{y}$, such that $(Y, \Gamma)$ is a proximal system. Then the extension $\pi$ is point distal. In particular, a metric tame minimal system admitting an invariant probability measure is point distal.

Proof. We will prove that the condition in Theorem 1.9 holds; i.e. that every $S$ set in $R_{\pi}$ is minimal. So let $W \subset R_{\pi}$ be an $S$-set. For $y \in Y$ we write $W_{y}=$ $W \cap\left(\pi^{-1}(y) \times \pi^{-1}(y)\right)$.

By Corollary 2.3 there is a dense $G_{\delta}$ subset $Y_{\text {so }} \subset Y$ such that for each $y \in Y_{\text {so }}$ the projection maps $\mathbf{p}_{i}: W_{y} \rightarrow \pi^{-1}(y)(i=1,2)$ are semiopen. Next recall that the set $W_{t r}$ of transitive points of $W$ forms a dense $G_{\delta}$ subset of $W$. By the Ulam theorem there is a dense $G_{\delta}$ subset $Y_{t r} \subset Y$ such that for each $y \in Y_{t r}, W_{t r} \cap\left(\pi^{-1}(y) \times \pi^{-1}(y)\right)$ is a dense $G_{\delta}$ subset of $W_{y}$. Thus, the set $Y_{s o} \cap Y_{t r} \cap Y_{f s}$ is also a dense $G_{\delta}$ subset of $Y$ (see Lemma 1.2.4 for the definition of $Y_{f s}$ ). Pick a point $y$ in this intersection.

Let $v$ be a minimal idempotent in $E(X, \Gamma)$ such that $v y=y$. By Lemma 4.2.4 $v X \subset \pi^{-1}(y)$ is a residual subset of $\pi^{-1}(y)$. As $y \in Y_{s o}$ we conclude that the sets $W \cap \mathbf{p}_{\mathbf{i}}{ }^{-1}(v X)(i=1,2)$ are residual subsets of $W_{y}$ (see Lemma 2.1). Now pick a point $\left(x, x^{\prime}\right)$ in the set

$$
W_{t r} \cap\left(\mathbf{p}_{\mathbf{1}}{ }^{-1}(v X) \cap \mathbf{p}_{\mathbf{2}}{ }^{-1}(v X)\right)
$$

(which is residual in $\left.W_{y}\right)$. We then have $v\left(x, x^{\prime}\right)=\left(v x, v x^{\prime}\right)=\left(x, x^{\prime}\right)$, so that $\overline{G\left(x, x^{\prime}\right)}$ is a minimal set. On the other hand, being a point in $W_{t r}$, we have $\overline{G\left(x, x^{\prime}\right)}=W$ and our proof is complete. For the last assertion, take $Y$ to be the trivial one point system.

\section{The STRUCtURE OF TAME METRIC Minimal DYNAMICAL SYSTEMS}

5.1. Definition. Let us call a minimal dynamical system $(X, \Gamma)$ standard if it has the following structure:

$$
X \stackrel{\pi}{\rightarrow} Y
$$

where $(Y, \Gamma)$ is a strongly proximal system and $\pi$ is a RIM extension.

The next theorem, from [15], is just a description of the RIM shadow diagram (1.6), associated to the map $X \rightarrow\{*\}$, the trivial one point system. The fact that, when $X$ is tame so is $\tilde{X}$, follows from Theorem 4.1 .

5.2. Theorem. Every minimal system $(X, \Gamma)$ has a standard extension $\tilde{X} \stackrel{\eta}{\rightarrow} X$ :

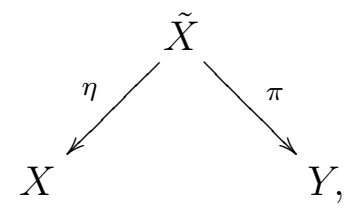

where $(Y, \Gamma)$ is a strongly proximal system, $\eta$ is a strongly proximal extension, and $\pi$ a RIM extension. If $(X, \Gamma)$ is tame then so is $(\tilde{X}, \Gamma)$.

We can now state and prove our main result. 
5.3. Theorem. Let $\Gamma$ be any group and $(X, \Gamma)$ a metric tame minimal system. Then there exists a commutative diagram

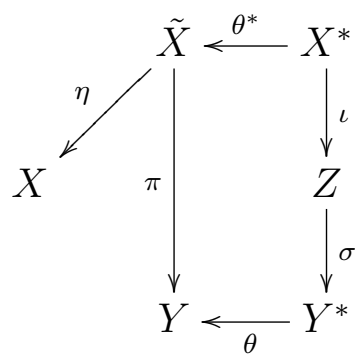

where

1. $\tilde{X}$ is metric minimal and tame.

2. $\eta$ is a strongly proximal extension.

3. $\pi$ is a RIM and point distal extension, with unique section $y \mapsto \nu_{y}$.

4. $(Y, \Gamma)$ is a strongly proximal system.

5. The maps $\theta, \theta^{*}$ and $\iota$ are almost one-to-one extensions.

6. $\sigma$ is an isometric extension.

Moreover, the extension $\pi^{*}=\sigma \circ \iota$ is a RIM extension with a unique section $y^{*} \mapsto \nu_{y^{*}}^{*}$. For each $y^{*} \in Y^{*}, \theta^{*}\left(\nu_{y^{*}}^{*}\right)=\nu_{\theta\left(y^{*}\right)}$ and the measure $\iota\left(\nu_{y^{*}}^{*}\right)$ is the Haar measure $\lambda_{y^{*}}$ on the fiber $\sigma^{-1}\left(y^{*}\right)$. The restriction $\iota:\left(\left(\pi^{*}\right)^{-1}\left(y^{*}\right), \nu_{y^{*}}^{*}\right) \rightarrow\left(\sigma^{-1}\left(y^{*}\right), \lambda_{y^{*}}\right)$ is a measure theoretical isomorphism.

Proof. 1. By Theorem 5.2 we have the RIM shadow diagram (5.1) with tame $\tilde{X}$. By Theorem 4.4 the RIM extension $\pi$ is point distal.

2. Next apply the structure theorem of point distal extensions, Theorem 1.8, and consider the first two stages of the corresponding AI tower

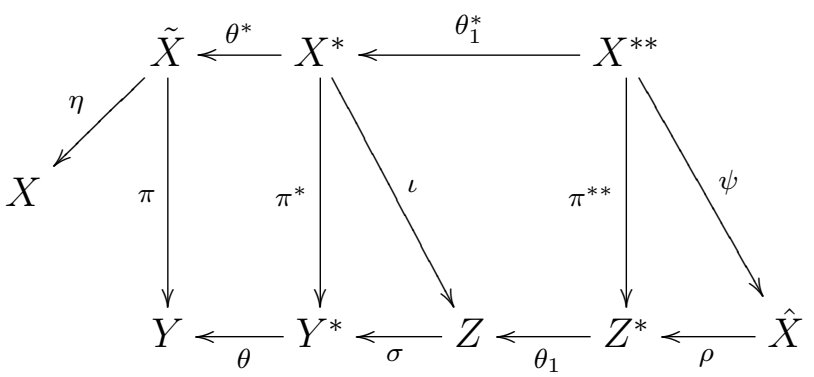

Thus

- the diagram

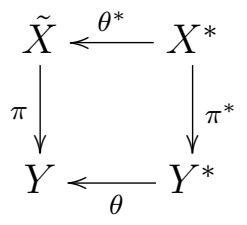

is the O-shadow diagram associated to $\pi$ (note that we no longer know that the system $X^{*}$ is tame),

- $\sigma$ is the largest intermediate isometric extension for $\pi^{*}$, 
- the diagram

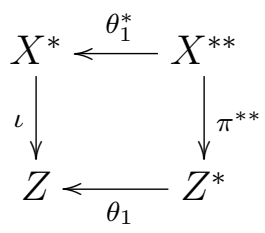

is the O-shadow diagram associated to $\iota$,

- and finally, $\rho: \hat{X} \rightarrow Z^{*}$ is the largest intermediate isometric extension for $\pi^{* *}$.

Note that if the map $\rho$ is an isomorphism; i.e. the largest isometric extension corresponding to $\pi^{* *}$ is trivial, then the AI tower for $\pi$ collapses to the diagram (5.2) which is exactly what we are after. Thus our goal is to show that indeed $\rho$ is necessarily an isomorphism.

3. In order to arrive at a contradiction, we now assume that $\rho$ is a nontrivial isometric extension. The map $\rho$, being isometric, is open RIM extension with unique section $z^{*} \mapsto \lambda_{z^{*}}$ (where $\lambda_{z^{*}}$ is the Haar measure on the homogeneous space of the associated compact group, see the diagram (1.2)).

Consider the subdiagram of (5.3)

$$
\hat{X} \stackrel{\rho}{\rightarrow} Z^{*} \stackrel{\theta_{1}}{\rightarrow} Z \stackrel{\sigma}{\rightarrow} Y^{*}
$$

We apply Proposition 3.5 (with $\hat{X}$ in the role of $X, Y^{*}$ in the role of $Y, \theta_{1}$ replacing $\theta$, and $\left.\phi=\sigma \circ \theta_{1} \circ \rho: \hat{X} \rightarrow Y^{*}\right)$. Let $y_{0}^{*} \in Y^{*}$ be the unique point with $u y_{0}^{*}=y_{0}^{*}$. Denote $\hat{X}_{0}=\phi^{-1}\left(y_{0}^{*}\right)$ and $X_{0}^{* *}=\psi^{-1}\left(\hat{X}_{0}\right)=\left(\pi^{*} \circ \theta_{1}^{*}\right)^{-1}\left(y_{0}^{*}\right)$.

Set $\mathfrak{M}_{m}\left(\hat{X}_{0}\right)=\left\{\Lambda(\nu): \nu \in \mathfrak{M}\left(Z_{0}^{*}\right)\right\}$. By Proposition 3.5, the set

$$
\hat{R}=\left\{\nu \in \mathfrak{M}\left(\hat{X}_{0}\right): \text { the orbit closure of } \nu \text { meets } \mathfrak{M}_{m}\left(\hat{X}_{0}\right)\right\}
$$

is a dense $G_{\delta}$ subset of $\mathfrak{M}\left(\hat{X}_{0}\right)$.

Set $\tilde{X}_{0}=\left(\theta^{*} \circ \theta_{1}^{*}\right)\left(X_{0}^{* *}\right)$. Also, set

$$
\begin{gathered}
\mathfrak{M}_{m}\left(X_{0}^{* *}\right):=\psi^{-1}\left(\mathfrak{M}_{m}\left(\hat{X}_{0}\right)\right) \text { and } \\
\mathfrak{M}_{m}\left(\tilde{X}_{0}\right):=\left(\theta^{*} \circ \theta_{1}^{*}\right)\left(\mathfrak{M}_{m}\left(X_{0}^{* *}\right)\right)=\left(\theta^{*} \circ \theta_{1}^{*}\right)\left(\psi^{-1}\left(\mathfrak{M}_{m}\left(\hat{X}_{0}\right)\right)\right) .
\end{gathered}
$$

Let

$$
R^{* *}:=\psi^{-1}(\hat{R})=\left\{\xi \in \mathfrak{M}\left(X_{0}^{* *}\right): \text { the orbit closure of } \xi \text { meets } \mathfrak{M}_{m}\left(X_{0}^{* *}\right)\right\}
$$

and

$$
R^{\prime}:=\left(\theta^{*} \circ \theta_{1}^{*}\right)\left(R^{* *}\right) \subset R
$$

where

$$
R=\left\{\xi \in \mathfrak{M}\left(\tilde{X}_{0}\right): \text { the orbit closure of } \xi \text { meets } \mathfrak{M}_{m}\left(\tilde{X}_{0}\right)\right\}
$$

By Lemma 2.5 the map $\psi: X^{* *} \rightarrow \hat{X}$ is semiopen and by Lemma 2.2 there is a dense $G_{\delta}$ subset $Y_{0}^{*} \subset Y^{*}$ such that $\psi:\left(\pi^{*} \circ \theta_{1}^{*}\right)^{-1}\left(y^{*}\right) \rightarrow \phi^{-1}\left(y^{*}\right)$ is semiopen for every $y^{*} \in Y_{0}^{*}$. By Lemma 3.1.2 we may and will assume that $y_{0}^{*} \in Y_{0}^{*}$, so that $\psi: X_{0}^{* *} \rightarrow \hat{X}_{0}$ is semiopen. Theorem 2.4 implies that $\psi: \mathfrak{M}\left(X^{* *}\right) \rightarrow \mathfrak{M}\left(\hat{X}_{0}\right)$ is semiopen as well. 
Now $\hat{R}$ is a dense subset of $\mathfrak{M}\left(\hat{X}_{0}\right)$ and Lemma 2.1 implies that $R^{* *}$ is a dense subset of $\mathfrak{M}\left(X_{0}^{* *}\right)$. Therefore $R^{\prime}$ is a dense subset of $\mathfrak{M}\left(\tilde{X}_{0}\right)$. From the definition of $R$ (5.4) it is easy to deduce that it is a $G_{\delta}$ set and because it contains $R^{\prime}$, it is in fact a dense $G_{\delta}$ subset of $\mathfrak{M}\left(\tilde{X}_{0}\right)$.

4. Recall that the system $(\tilde{X}, \Gamma)$ is tame and, by Theorem 4.1 so is $(\mathfrak{M}(\tilde{X}), \Gamma)$. Moreover we have $E(\tilde{X}, \Gamma)=E(\mathfrak{M}(\tilde{X}), \Gamma)$. In particular $u \in E(\mathfrak{M}(\tilde{X}), \Gamma)$, as a Baire 1 function, has a dense $G_{\delta}$ set of continuity points. Moreover, $C_{u}=C_{\overline{u \mathfrak{M}(\tilde{X})}}(u)$, the set of continuity points of $u$ restricted to the set $\overline{u \mathfrak{M}(\tilde{X})}$, is a dense $G_{\delta}$ subset of $\overline{u \mathfrak{M}(\tilde{X})}$ and, by Lemma 4.2.3,

$$
C_{u} \subset u \mathfrak{M}(\tilde{X})
$$

As $\rho$ and $\sigma$ are isometric extensions and $\theta_{1}$ is an almost one-to-one extension, it is easy to see that $\overline{u \hat{X}}=\hat{X}_{0}$. As $\psi$ is semiopen and a point distal extension, it follows that $\overline{u X^{* *}}=X_{0}^{* *}$ and therefore also that $\overline{u \tilde{X}}=\tilde{X}_{0}$. It follows that the collection of finite convex combinations of point masses picked from $u \tilde{X}$ forms a dense subset of $\mathfrak{M}\left(\tilde{X}_{0}\right)$. In turn, this implies that $\overline{u \mathfrak{M}(\tilde{X})}=\mathfrak{M}\left(\tilde{X}_{0}\right)$ and we conclude that $S:=C_{u} \cap R \subset u \mathfrak{M}(\tilde{X})$ is a dense $G_{\delta}$ subset of $\mathfrak{M}\left(\tilde{X}_{0}\right)$.

Now if $\nu \in S$ then $u \nu=\nu$ and, $u$ being a minimal idempotent, the closure of the $\Gamma$ orbit of $\nu$ in $\mathfrak{M}(\tilde{X})$ is a minimal set. On the other hand, $\nu$ being also an element of $R$, this orbit closure meets $\mathfrak{M}_{m}\left(\tilde{X}_{0}\right)$. We conclude that $\overline{\Gamma \nu}$ is contained in $\mathfrak{M}_{m}(\tilde{X})$. In particular $\nu \in \mathfrak{M}_{m}(\tilde{X}) \cap \mathfrak{M}\left(\tilde{X}_{0}\right)=\mathfrak{M}_{m}\left(\tilde{X}_{0}\right)$ and we conclude that $S \subset \mathfrak{M}_{m}\left(\tilde{X}_{0}\right)$, whence the equality

$$
\mathfrak{M}_{m}\left(\tilde{X}_{0}\right)=\mathfrak{M}\left(\tilde{X}_{0}\right)
$$

5. Given a point $\tilde{x} \in \tilde{X}_{0}$, the corresponding point mass $\delta_{\tilde{x}} \in \mathfrak{M}\left(\tilde{X}_{0}\right)$ must have, by (5.5), a preimage in $\mathfrak{M}_{m}\left(X_{0}^{* *}\right)$, say $\left(\theta^{*} \circ \theta_{1}^{*}\right)(\xi)=\delta_{\tilde{x}}$ with $\xi \in \mathfrak{M}_{m}\left(X_{0}^{* *}\right)$. For $\tilde{x}$ with $\left(\theta^{*} \circ \theta_{1}^{*}\right)^{-1}(\tilde{x})=\left\{x^{* *}\right\}$ a singleton, we must have $\xi=\delta_{x^{* *}} \in \mathfrak{M}_{m}\left(X_{0}^{* *}\right)$ and therefore $\psi_{*}\left(\delta_{x^{* *}}\right)=\delta_{\hat{x}} \in \mathfrak{M}_{m}\left(\hat{X}_{0}\right)$ with $\hat{x}=\psi\left(x^{* *}\right)$. By the definition of $\mathfrak{M}_{m}\left(\hat{X}_{0}\right)$ there exists a measure $\zeta \in \mathfrak{M}\left(Z_{0}^{*}\right)$ with

$$
\delta_{\hat{x}}=\Lambda(\zeta)=\int_{Z^{*}} \lambda_{z^{*}} d \zeta\left(z^{*}\right) .
$$

This clearly implies that the measure $\zeta$ is a point mass, say $\zeta=\delta_{z^{*}}$ and that the measure $\lambda_{z^{*}}$ - which is the Haar measure on the homogeneous space which forms the fiber $\rho^{-1}\left(z^{*}\right) \subset \hat{X}-$ is also a degenerate point mass. That is, the isometric extension $\rho$ is in fact an isomorphism. As we observed above, the collapse of $\rho$ implies the collapse of the entire AI tower for $\pi$, and the diagram (5.2) is obtained.

6. By Lemma 1.3 the section for $\pi^{*}$ has the form $y^{*} \mapsto \nu_{y^{*}}^{*}=\nu_{\theta\left(y^{*}\right)} \times \delta_{\theta\left(y^{*}\right)}$, whence $\theta^{*}\left(\nu_{y^{*}}^{*}\right)=\nu_{\theta\left(y^{*}\right)}$. Now the extension $\sigma: Z \rightarrow Y^{*}$, being isometric, admits a unique section $y^{*} \mapsto \lambda_{y^{*}}$, where $\lambda_{y^{*}}$ is the Haar measure on the homogeneous space $\sigma^{-1}\left(y^{*}\right)$. It follows that $\iota\left(\nu_{y^{*}}^{*}\right)$ is the Haar measure $\lambda_{y^{*}}$ on the fiber $\sigma^{-1}\left(y^{*}\right)$.

From the construction of the O-shadow diagram it follows that the map

$$
\theta^{*}:\left(\pi^{*}\right)^{-1}\left(y^{*}\right) \rightarrow \pi^{-1}(y)
$$


(with $y=\theta\left(y^{*}\right)$ ) is a homeomorphism. Therefore we can study the nature of the map $\iota:\left(\left(\pi^{*}\right)^{-1}\left(y^{*}\right), \nu_{y^{*}}\right) \rightarrow\left(\sigma^{-1}\left(y^{*}\right), \lambda_{y^{*}}\right)$ via its pushforward

$$
\iota \circ\left(\theta^{*}\right)^{-1}:\left(\pi^{-1}(y), \nu_{y}\right) \rightarrow\left(\sigma^{-1}\left(y^{*}\right), \lambda_{y^{*}}\right) .
$$

Let $v y^{*}=y^{*}$ for a minimal idempotent $v$. Then also $v y=y$ and, $\tilde{X}$ being tame, we have $\nu_{y}(v \tilde{X})=1$ by Lemma 4.2.2. We disintegrate $\nu_{y^{*}}^{*}$ over $\lambda_{y *}$

$$
\nu_{y^{*}}^{*}=\int_{\sigma^{-1}\left(y^{*}\right)} \xi_{z} d \lambda_{y *}(z),
$$

and correspondingly $\nu_{y}$ over $\lambda_{y}$, say

$$
\nu_{y}=\int \xi_{\omega} d \lambda_{y}(\omega)
$$

Now in the first disintegration, for $\lambda_{y^{*}}$ almost every $z$, the measure $\xi_{z}$ is concentrated on $\iota^{-1}(z)$, which consists of pairwise proximal points ( $\iota$ being a proximal extension). On the other hand, any two points in $v \tilde{X}$ are distal. We therefore conclude that for $\lambda_{y^{*}}$ almost every $z$, the measure $\xi_{\omega}$, and therefore also $\xi_{z}$, is a point mass. This means that indeed $\iota:\left(\left(\pi^{*}\right)^{-1}\left(y^{*}\right), \nu_{y^{*}}\right) \rightarrow\left(\sigma^{-1}\left(y^{*}\right), \lambda_{y^{*}}\right)$ is a measure theoretical isomorphism.

Since the Haar measures section is unique we conclude that the section $y^{*} \mapsto \nu_{y^{*}}^{*}$ is unique. Finally, by Lemma 1.3 , also $y \mapsto \nu_{y}$ is unique.

5.4. Corollary. With notations as in Theorem 5.3

1. If the RIM extension $\pi$ happens to be open then the diagram (5.2) reduces to the simplified form

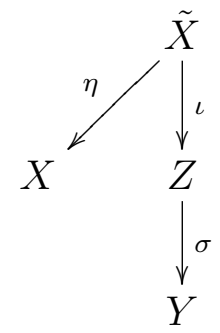

with $\pi=\sigma \circ \iota$.

2. If the system $(X, \Gamma)$ admits an invariant probability measure $\mu$ then $X$ is almost automorphic, i.e. it has the form $X \stackrel{\iota}{\rightarrow} Z$, where $Z$ is equicontinuous and $\iota$ is almost one-to-one. Moreover, $\mu$ is unique and the map $\iota$ is a measure theoretical isomorphism $\iota:(X, \mu, \Gamma) \rightarrow(Z, \lambda, \Gamma)$, where $\lambda$ is the Haar measure on the homogeneous space $Z$.

3. Thus, when $\Gamma$ is amenable this latter situation is the rule.

Concerning the first assertion in Corollary 5.4 we pose the following.

5.5. Problem. Is a RIM homomorphism $\pi: X \rightarrow Y$ of minimal metric systems necessarily open ? Is this true when, in addition, we assume that $X$ is tame?

5.6. Remark. In the situation described in Corollary 5.4.2, the set $X_{0}=\{x \in X$ : $\left.\iota^{-1}(\iota(x))=\{x\}\right\}$ is a dense $G_{\delta}$ and $\Gamma$-invariant subset of $X$ and thus has $\mu$ measure either zero or one. In [28, Section 11] Kerr and Li construct a minimal Toeplitz cascade (i.e. a $\mathbb{Z}$-system) which is tame and not null. In [21, Remark 5.2] I claimed 
that this example can be made not regular in the sense that the densities of the periodic parts converge to $d<1$. As for such non-regular systems $\mu\left(X_{0}\right)=0$, this would show that the unique invariant measure of a minimal tame system need not be supported by the set $X_{0}$ where $\pi$ is 1-1. Unfortunately the argument I had in mind when claiming that the Kerr-Li example can be made nonregular was flawed. Thus the following basic question is still open.

5.7. Problem. Let $(X, \Gamma)$ be a metric minimal tame system. Suppose that $(X, \Gamma)$ admits a (necessarily unique) invariant probability measure $\mu$. Let $X \stackrel{\iota}{\rightarrow} Z$ be its maximal equicontinuous factor (so that $\iota$ is an almost one-to-one extension). Let $X_{0} \subset X$ be the dense $G_{\delta}$ subset of $X$ defined by $X_{0}=\left\{x \in X: \iota^{-1}(\iota(x))=\{x\}\right\}$. Is it necessarily the case that $\mu\left(X_{0}\right)=1$ ?

5.8. Remark. On the other hand, one may ask whether every metric minimal almost automorphic system $(X, \Gamma)$, with $X \stackrel{\iota}{\rightarrow} Z$ being its maximal equicontinuous factor (so that $\iota$ is an almost one-to-one extension), which admits a unique invariant measure $\mu$ such that $\mu\left(X_{0}\right)=1$, is necessarily tame ? The answer here is no. We can construct a minimal subshift $X \subset\{0,1\}^{\mathbb{Z}}$ as above, with $Z$ being an irrational rotation of the circle, yet $(X, \sigma)$ ( $\sigma$ denoting the shift) is not tame. We omit the details which will be published elsewhere.

5.9. Remark. The following corollary of Theorem 5.3 was suggested by the referee. The notion of mean equicontinuity was studied in [30] and [7]. (A $\mathbb{Z}$-system is mean equicontinuous if for every $\epsilon>0$ there is $\delta>0$ such that if $d(x, y)<\delta$ then $d\left(T^{i} x, T^{i} y\right)<\epsilon$ for all $i$ except for a set of density $<\epsilon$.) Combining Theorem 2.1 of [7] and Theorem 5.3 we deduce the following:

5.10. Corollary. A minimal tame $\mathbb{Z}$-system is mean equicontinuous.

\section{EXAMPLES}

We illustrate our main theorem, Theorem 5.3, with some basic examples.

- The classical Sturmian $\mathbb{Z}$ dynamical system $(X, T)$ (where $T: X \rightarrow X$ is the homeomorphism which generate the $\mathbb{Z}$ action) is minimal and tame. This is an almost automorphic system with $\theta: X \rightarrow Z$, an almost one-to-one factor, the projection of $X$ onto its largest equicontinuous factor $Z$, the dyadic adding machine. See [22].

- Every null dynamical system is tame; see [27] and [28]. (A system is null if it has zero sequence topological entropy with respect to every subsequence $\left.n_{i} \nearrow \infty.\right)$

- The action of $G=G L(d, \mathbb{R})$ (and hence of any subsgroup $\Gamma<G$ ) on the projective space $\mathbb{P}^{d-1}$, comprising the lines through the origin in $\mathbb{R}^{n}$, is tame (see the appendix below). The same is true for the action of $G$ on $S^{d-1}$, identified with the space of rays emanating from the origin. We have a natural two-to-one map $\sigma: S^{d-1} \rightarrow \mathbb{P}^{d-1}$. This factor map corresponds to the diagram (5.6) with $X=\tilde{X}=Z=S^{d}$ and $Y=\mathbb{P}^{d-1}$. See [14], [9] and [1].

- The action of the group $G=\operatorname{Homeo}\left(\mathrm{S}^{1}\right)$ (and hence of any subsgroup $\Gamma<G$ ) on the circle $S^{1}$ is tame. See [24]. 
- Our next example will demonstrate the necessity for the presence of the extension $\eta$ in the diagram (5.6). It is an elaboration of an example of Furstenberg and Glasner, see [18]. Let $G$ be the closed subgroup of the Lie group $G L(4, \mathbb{R})$ consisting of all $4 \times 4$ matrices of the form

$$
\left(\begin{array}{cc}
A & 0 \\
0 & B
\end{array}\right) \text { and }\left(\begin{array}{cc}
0 & A \\
B & 0,
\end{array}\right)
$$

with $A, B \in G L(2, \mathbb{R})$. We let $G$ act on the subspace $X$ of the projective space $\mathbb{P}^{3}$ consisting of the disjoint union of the two one dimensional projective spaces $\mathbb{P}^{1}$, which are naturally embedded in $\mathbb{P}^{3}$, the quotient space of $\mathbb{R}^{4}=\mathbb{R}^{2} \times \mathbb{R}^{2}$. Call these two copies $X_{1}$ and $X_{2}$ respectively. By [1] this action is tame.

There is a natural projection from $(X, G)$ onto the two points $G$-system $\left(\left\{X_{1}, X_{2}\right\}, G\right)$. It is now easy to establish the remaining assertions of the following:

Claim 1: The $G$-system $(X, G)$ is minimal and tame. It admits the isometric factor which is the "flip" on two points and the map from $X$ onto $\left(\left\{X_{1}, X_{2}\right\}, G\right)$ is a strongly proximal extension. However, the system $(X, G)$ admits no nontrivial proximal factor.

Let $Y$ be the "quasifactor" of $(X, G)$ defined by:

$$
Y=\left\{\frac{1}{2}\left(\delta_{x_{1}}+\delta_{x_{2}}\right): x_{i} \in X_{i}, i=1,2\right\} \subset \mathfrak{M}(X) .
$$

Again it is easy to check that the system $(Y, G)$ is minimal and strongly proximal $G$-system.

Next let

$$
\tilde{X}=\left\{\left(x_{i}, \frac{1}{2}\left(\delta_{x_{1}}+\delta_{x_{2}}\right)\right): x_{i} \in X_{i}, i=1,2\right\} \subset X \times \mathfrak{M}(X)
$$

Claim 2: $\tilde{X}$ is minimal and tame. Let $\eta: \tilde{X} \rightarrow X$ and $\sigma: \tilde{X} \rightarrow Y$ denote the projections from $\hat{X}$ onto its two components. Then the diagram (5.6) (with $Z=\tilde{X}$ and $\iota$ the identity map) is the canonical standard extension describing the structure of the minimal tame system $X$.

\section{Appendix: Borel's Density theOREM}

In this short section we will rewrite Furstenberg's proof of Borel's density theorem [14], in terms of tame systems and enveloping semigroups.

As we will show next the action of $G=S L_{d}(\mathbb{R})$ on the projective space $\mathbb{P}^{d-1}$ $(d \geq 2)$ is tame. This is also true for the $G$ action on $S^{d-1}$. In both cases the enveloping semigroup is a Rosenthal compactum but not metrizable. In the case of the projective space it is not even first countable (see [1]). Thus, these systems are tame but not HAE.

For a non-zero vector $v \in \mathbb{R}^{d}$ we let $\bar{v}$ denote its image in $\mathbb{P}^{d-1}$. Similarly, for $g \in G$ we let $\bar{g}$ denote its image in $E\left(\mathbb{P}^{d-1}, S L_{d}(\mathbb{R})\right)$.

7.1. Lemma. The action of $G L_{d}(\mathbb{R})$ on $\mathbb{P}^{d-1}$ is tame. 
Proof. Let $g_{n}$ be a sequence of matrices in $G L_{d}(\mathbb{R})$. Given any subspace $W \subset \mathbb{R}^{d}$, by passing to a subsequence and by choosing appropriate scalars $\lambda_{n}$, we can assume that $\lambda_{n} g_{n} \rightarrow h_{W}$, a non-zero linear map from $W$ into $\mathbb{R}^{d}$. For $v \notin \operatorname{ker}\left(h_{W}\right)$ we have $\overline{\lambda_{n} g_{n}(v)} \rightarrow \overline{h_{W}(v)}$.

Now define $W_{0}=\mathbb{R}^{d}, W_{1}=\operatorname{ker}\left(h_{W_{0}}\right)$, and repeat this procedure with $W_{1}$ to obtain $h_{W_{1}}$ which maps $W_{1}$ onto a subspace of $\mathbb{R}^{d}$. Proceeding by induction (till we have a trivial kernel) we finally obtain a subsequence such that $\overline{g_{n_{i}}} \rightarrow p \in E\left(\mathbb{P}^{d-1}, S L_{d}(\mathbb{R})\right)$. This shows that $\left(\mathbb{P}^{d-1}, G\right)$ is tame.

Note that for $W=\mathbb{R}^{d}$ the assumptions $g_{n} \in S L_{d}(\mathbb{R})$ and $\left\|g_{n}\right\| \rightarrow \infty$, imply that $\operatorname{dim} \operatorname{ker} h_{W} \geq 1$. Thus, in this case the image of $p$ is a finite union of projective sub-verieties of dimension $<d-1$ (a quasi-sub-veriety).

7.2. Definition. A pair of groups $(G, H)$ is called a Borel pair if $G$ is a minimally almost periodic (m.a.p.) group and $H$ is a closed subgroup of $G$ such that the quotient space $G / H$ supports a finite $G$-invariant measure.

7.3. Theorem (Borel's density theorem). Let $(G, H)$ be a Borel pair and $\pi$ a finitedimensional representation of $G$ on a space $V$. If $W$ is a $\pi(H)$-invariant subspace, it is also $\pi(G)$-invariant.

Proof. Suppose first that $\operatorname{dim}(W)=1$, say $W=\mathbb{R} v$, so that $x_{0}=\bar{v} \in \mathbb{P}(V)$. Then, the map $g \mapsto \overline{g v}=g x_{0}$, from $G / H$ into $\mathbb{P}(V)$, sends the invariant measure on $G / H$ to an invariant measure, say $\mu$, on $\mathbb{P}(V)$. Let $Y$ be the support of $\mu$. Clearly $x_{0} \in Y$ and $Y$ is a closed $G$-invariant subset. Let $u \in E(\mathbb{P}(V), G)$ be a minimal idempotent. Then (by tameness; see Theorem 4.1) $u_{*} \mu=\mu$ and it follows that $\mu(u \mathbb{P}(V))=1$. By tameness there is a sequence $g_{n} \in G$ with $\overline{g_{n}} \rightarrow u$. The range of $u$ can not be all of $\mathbb{P}(V)$ since this would mean that the enveloping semigroup is actually a group and therefore, that the action is distal; however, this possibility is ruled out by the m.a.p. property of $G$. Thus, the set $u \mathbb{P}(V)$ is a quasi-sub-veriety (finite union of proper sub-varieties). In particular it is closed, whence $Y \subset u \mathbb{P}(V)$.

Let $L=\bigcup L_{i}$ be a minimal projective quasi-sub-variety containing $Y$. Then $L$ is closed and $G$-invariant and $G$ must permute its components. By m.a.p. the permutations are all the identity permutations. Thus each $L_{i}$ is $G$-invariant.

If the $G$-action on $L_{i}$ is not trivial then, again we conclude that $u L_{i} \subsetneq L_{i}$, which contradicts the minimality of $L$. Thus $G$ acts on $L$ as the identity and, in particular, $x_{0} \in Y \subset L$ is a $G$-fixed point.

The general case is reduced to the 1-dimensional one using exterior products.

We refer the reader to [3] and [14] where it is shown how from this theorem one easily deduces the remaining results of [3].

\section{REFERENCES}

1. E. Akin, Enveloping linear maps, in: Topological dynamics and applications, Contemporary Mathematics 215, 1998, pp. 121-131.

2. J. Auslander, Minimal Flows and their Extensions, Mathematics Studies 153, Notas de Matemática, 1988.

3. A. Borel, Density properties of certain subgroups of semisimple groups without compact components, Ann. of Math. (2) 72(1960), 179-188. 
4. J. Bourgain, D. H. Fremlin and M. Talagrand, Pointwise compact sets of Baire-measurable functions, Amer. J. Math., 100 (1978), 845-886.

5. I. U. Bronstein, A characteristic property of PD-extensions, Bul. Akad. Stiimce RSS Moldoven, 3, (1977), 11-15 (Russian).

6. S. Z. Ditor and L. Q. Eifler, Some open mapping theorems for measures, Trans. Amer. Math. Soc., 164, (1972), 287-293.

7. T. Downarowicz, and E. Glasner, Isomorphic extensions and applications, Topol. Methods Nonlinear Anal. 48, (2016), 321-338.

8. R. Ellis, A semigroup associated with a transformation group, Trans. Amer. Math. Soc., 94, (1960), 272-281.

9. 635-660.

10. _ Lectures on Topological Dynamics, W. A. Benjamin, Inc. , New York, 1969.

11. _ The Veech structure theorem, Trans. Amer. Math. Soc. 186, (1973), 203-218 (1974).

12. R. Ellis, E. Glasner and L. Shapiro, Proximal-Isometric flows, Advances in Math., 17, (1975), 213-260.

13. H. Furstenberg, The structure of distal flows, American J. of Math., 85, (1963), 477-515.

14. _ A note on Borel's density theorem, Proc. Amer. Math. Soc. 55, (1976), 209-212.

15. E. Glasner, Relatively invariant measures, Pacific J. of Math., 58, (1975), 393-410.

16. _ Proximal flows, Lecture Notes in Math., 517, Springer-Verlag, 1976.

17. __ Distal and semisimple affine flows, Amer. J. Math., 109, (1987), 115-131.

18. __ Quasifactors of minimal systems, Topol. Methods Nonlinear Anal. 16, (2000), 351370.

19. Structure theory as a tool in topological dynamics, Descriptive set theory and dynamical systems, LMS Lecture note Series 277, Cambridge University Press, Cambridge, 2000, 173-209.

20. __ On tame dynamical systems, Colloq. Math., 105 (2006), 283-295.

21. _ The structure of tame minimal dynamical systems, Ergod. Th. \& Dynam. Sys. 27, (2007), 1819-1837.

22. E. Glasner and M. Megrelishvili, Hereditarily non-sensitive dynamical systems and linear representations, Colloq. Math., 104 (2006), no. 2, 223-283.

23. _ Eventual nonsensitivity and tame dynamical systems, arXiv:1405.2588.

24. _ Circularly ordered dynamical systems, a preprint.

25. E. Glasner, M. Megrelishvili and V.V. Uspenskij, On metrizable enveloping semigroups, Israel J. of Math. 164 (2008), 317-332.

26. W. Huang, Tame systems and scrambled pairs under an abelian group action, Ergod. Th. Dynam. Sys., 26, (2006), 1549-1567.

27. W. Huang, S. M. Li, S. Shao, and X. Ye, Null systems and sequence entropy pairs, Ergod. Th. Dynam. Sys., 23, (2003), 1505-1523.

28. D. Kerr and H. Li, Independence in topological and $C^{*}$-dynamics, Math. Ann. 338 (2007), 869-926.

29. A. Köhler, Enveloping semigroups for flows, Proceedings of the Royal Irish Academy, 95A, (1995), 179-191.

30. Jian Li, S. Tu and X. Ye, Mean equicontinuity and mean sensitivity, Ergod. Th. and Dynam. Sys., 35, (2015), 2587-2612.

31. D. C. McMahon, Weak mixing and a note on the structure theorem for minimal transformation groups, Illinois J. of Math., 20, (1976), 186-197.

32. W. A. Veech, Point-distal flows, Amer. J. Math. 92, (1970), 205-242.

33. _ Topological dynamics, Bull. Amer. Math. Soc., 83, (1977), 775-830.

34. J. de Vries, Elements of Topological Dynamics, Kluwer Academic Publishers, 1993.

35. J. van der Woude, Characterizations of $H(P I)$ extensions, Pacific J. Math., 120, (1985), 453-467. 
Department of Mathematics, Tel-Aviv University, Tel Aviv, Israel E-mail address: glasner@math.tau.ac.il 